\section{Research Square}

\title{
Age-Related Changes in the Gut Microbiota Promote Atrial Fibrillation
}

\section{Yun Zhang}

the first affiliated hospital of harbin medical university

\section{Song Zhang}

First Affiliated Hospital of Harbin Medical University

\section{Bolin Li}

Xi'an Jiaotong University Medical College First Affiliated Hospital

\section{Yingchun Luo}

First Affiliated Hospital of Harbin Medical University

\section{Yongtai Gong}

First Affiliated Hospital of Harbin Medical University

\section{Xuexin Jin}

First Affiliated Hospital of Harbin Medical University

\section{Jiawei Zhang}

First Affiliated Hospital of Harbin Medical University

\section{Yun Zhou}

First Affiliated Hospital of Harbin Medical University

\section{Xiaozhen Zhuo}

Xi'an Jiaotong University Medical College First Affiliated Hospital

\section{Zixi Wang}

Xi'an Jiaotong University Medical College First Affiliated Hospital

\section{Xinbo Zhao}

First Affiliated Hospital of Harbin Medical University

\section{Xuejie Han}

First Affiliated Hospital of Harbin Medical University

\section{Yunlong Gao}

First Affiliated Hospital of Harbin Medical University

Hui Yu

First Affiliated Hospital of Harbin Medical University

\section{Desen Liang}

First Affiliated Hospital of Harbin Medical University

\section{Shiqi Zhao}

First Affiliated Hospital of Harbin Medical University 
First Affiliated Hospital of Harbin Medical University

\section{Dingyu Wang}

First Affiliated Hospital of Harbin Medical University

\section{Wei Xu}

First Affiliated Hospital of Harbin Medical University

\section{Guangjin Qu}

First Affiliated Hospital of Harbin Medical University

\section{Wanlan Bo}

First Affiliated Hospital of Harbin Medical University

\section{Dan Li}

First Affiliated Hospital of Harbin Medical University

\section{Yue Wu}

First Affiliated Hospital of Harbin Medical University

\section{Yue Li ( $\sim$ ly99ly@hrbmu.edu.cn )}

the first affiliated hospital of harbin medical university https://orcid.org/0000-0002-2353-4078

\section{Research}

Keywords: Atrial fibrillation, Aging, Gut microbiota, LPS, TLR-4, NLRP3-inflammasome

Posted Date: August 4th, 2020

DOI: https://doi.org/10.21203/rs.3.rs-51069/v1

License: (c) (1) This work is licensed under a Creative Commons Attribution 4.0 International License. Read Full License 


\section{Abstract}

Background: Aging is the most significant contributor to the increasing prevalence of atrial fibrillation (AF). The gut microbiota dysbiosis is involved in age-associated diseases. However, whether ageassociated gut microbial dysbiosis contributes to AF is still unknown. The aim of this study was to evaluate the effect of gut microbiota on the susceptibility of aging-induced AF and to elucidate the underlying mechanisms.

Results: The gut microbiota profiling of fecal samples in aged (22-24 months old) and young (2-3 months old) rats was performed by $16 \mathrm{~S}$ ribosomal RNA gene analysis. A rat model of fecal microbiota transplantation (FMT) was established for analyzing the possible role of age-associated gut microbial dysbiosis in AF. Here, we found that aging process led to marked shift of the microbiota spectrum. The microbiota in young rats following FMT resembled that of aged microbiota and transmitted the increased AF susceptibility by elevation of circulating lipopolysaccharide (LPS). The mechanism for LPS-driven atrial pro-arrhythmic action was dependent on the activation of atrial nucleotide binding and oligomerization domain-like receptor family pyrin domain-containing (NLRP3)-inflammasome. Inhibition of inflammasome by MCC950 resulted in lower atrial fibrosis and AF susceptibility. In addition, atrial fibrosis, plasma LPS concentration, plasma glucose to oral glucose tolerance test (OGTT), intestinal permeability, and gut pathology were significantly increased in elderly human subjects. Finally, altering the microbiota in aged recipient to resemble that of young restored the intestinal barrier dysfunction and LPS and impaired glucose tolerance, and the worse outcomes of aged dysbiosis on atrial fibrosis and AF susceptibility were abrogated.

Conclusions: These data suggest that age-associated microbial dysbiosis induces circulating LPS and impairs glucose tolerance, and thereby promotes AF susceptibility through enhanced activity of atrial NLRP3-inflammasome.

\section{Introduction}

Atrial fibrillation (AF) is the most common cardiac arrhythmia in clinical practice and it can cause substantial morbidity and mortality[1,2]. The incidence of AF rises steadily with increasing age. However, the complex complications and high recurrence rate in aged patients often hinder clinical AF treatment and prevention. Better understanding of aging-related AF and discovery of novel therapeutic strategies are urgently needed.

Gut microbiomes and their metabolites including the lipopolysaccharide (LPS), bile acids, trimethylamine $\mathrm{N}$-oxide (TMAO), and short-chain fatty acids, have been implicated in aging-related cardiovascular diseases[3-5]. It has been reported that the gut microbiota composition of older human differs from that of young one with decreased Bifidobacterium and Firmicutes and increased Bacteroidetes and Enterobacteriaceae, which is referred to gut dysbiosis in elders[6]. The age-associated gut microbiota dysbiosis could accelerate the rate of aging leading to the "inflamm-aging" state in the host[7, 8]. Besides, 
the increased levels of inflammatory cytokines have been found in AF patients[9], indicating that inflammation is closely linked to the development of $\mathrm{AF}[10,11]$. However, how age-associated microbial dysbiosis and the associated "inflamm-aging" status promote AF remains unclear and merits experimental elucidation.

Lipopolysaccharides (LPS) are consisted of a hydrophobic lipid A and a hydrophilic carbohydrate core with polysaccharide 0-antigen, and which belongs to cell wall structural component of Gram-negative (e.g. E. coli)[12]. LPS is a potent trigger of systemic inflammation in host and increased with aging process, suggesting that LPS is an important mediator in the development of "inflamm-aging"[5]. An increased serum LPS levels are prediction of adverse events in AF patients has been reported[4]. Indeed, LPS can translocate from the intestine by either paracellular or transcellular mechanisms to enter circulation and quickly recognized by the toll-like receptor (TLR)4[13]. TLR4 activates intracellular signaling cascades that results in the translocation of nuclear factor kappa B (NF-kB) and mediation of numerous pro-inflammatory genes expression. Thus, we speculated that gut derived-LPS had an implication on increased of age-related AF susceptibility, which may cause by alterations of aged dysbiosis-mediates systemic inflammation.

In the current study, we explored the causal relationship between age-associated microbiota dysbiosis and AF in a rat model of FMT. Our results showed that aged dysbiosis elevated LPS level and impaired glucose tolerance, thereby promoting aging-related AF through activating atrial NLRP3-inflammasome. The results obtained from plasma samples and atria and intestine tissues of human subjects confirmed the main findings of our animal study.

\section{Methods}

\section{Human Studies.}

The study protocol was approved by the Research Ethics Committees of The First Affiliated Hospital of Harbin Medical University (Harbin, Heilongjiang, China) and The First Affiliated Hospital of Xi'an Jiaotong University (Xi'an, Shaanxi, China) and performed in accordance with the Declaration of Helsinki. The participants all provided written informed consent. We retrospectively reviewed the oral glucose tolerance test (OGTT) records of the patients $(n=12,012)$ of Biobank at The First Affiliated Hospital of Xi'an Jiaotong University between January 2018 and September 2019. The clinical information was shown in Supplemental Table-1(Additional File 1). In addition, the following samples were collected from the qualified participants. First, the plasma samples of patients (18-75 years; $n=1,152)$ who underwent physical examination in The First Affiliated Hospital of Harbin Medical University between September 2019 and November 2019 were collected. The clinical information was shown in Supplemental Table-2 (Additional File 1). Second, the normal colon tissue samples from young (18-40 years; $n=10$ ) and old patients (over 65 years; $n=10$ ) who underwent the radical operation for left hemicolon cancer in The First Affiliated Hospital of Harbin Medical University were collected. The clinical information was shown in

Supplemental Table-3 (Additional File 1). Finally, the right atrial appendages of young (18 40 years; $n=6)$ 
and elderly (over 65 years; $n=6$ ) patients who underwent open-heart surgery for valve replacement in The First Affiliated Hospital of Harbin Medical University were collected. The clinical information was shown in Supplemental Table-4 (Additional File 1).

The general exclusion criteria of patients were a known history of diabetes (except OGTT analysis), treatment with antibiotics or probiotics within the past three months, or cold within the past month. In addition, other specific exclusion criteria were also applied. First, patients who had medical history of cancer, inflammatory bowel disease major gastrointestinal surgery within the past five years, invasive medical intervention within the past three months, or significantly altered diet compositions in the week of blood collection were excluded. Second, patients who had other intestinal inflammatory diseases except for colon cancer and major gastrointestinal surgery within the past five years were excluded. Finally, patients with end-stage heart failure, cancer, or inflammatory bowel diseases were also excluded.

\section{Experimental Animals.}

The animal experiments in this study were conducted in accordance with the Guide for the Care and Use of Laboratory Animals and approved by the Institutional Animal Care and Use Committee at the Harbin Medical University. Both aged male Sprague Dawley (SD) rats (22-24 months old) and young male SD rats (2-3 months old) were purchased from Beijing Vital River Laboratory Animal Technology Co, Ltd (Beijing, China). The rats were individually housed under 12:12-h light-dark cycles and fed with food and water available ad libitum. In anti-lipopolysaccharide (LPS) experiments, rats received oral LPS-RS (a potent LPS antagonist; InvivoGen, CA, USA) at $1 \mathrm{mg} / \mathrm{Kg}$ once per week for six weeks. In anti-NLRP3 experiments, rats received MCC950 (a small-molecule selective inhibitor of NLRP3; Sigma, San Jose, CA, USA; cat. no.: PZ0280-5MG) via intraperitoneal injection at $15 \mathrm{mg} / \mathrm{Kg}$ once per week for six weeks.

\section{Electrophysiological study.}

Atrial fibrillation (AF) was induced with essentially the same protocol as described previously in detail[14]. In brief, rats were anesthetized with $1 \%$ sodium pentobarbital $(30 \mathrm{mg} / \mathrm{kg})$ through peritoneal injection. After open-chest surgery, a 1.9-F octapolar catheter (Transonic Systems Inc., New York, USA) was placed on the right atrium to deliver programmed stimuli. To assess the inducibility of atrial arrhythmias, $50-\mathrm{Hz}$ burst pacing was applied for $3 \mathrm{~s}$ with 12 bursts separated by a 2-s interval. AF was defined as $>1 \mathrm{~s}$ of irregular atrial electrograms (>800 bmp) with irregular ventricular response. AF duration was defined as the mean duration of all $\mathrm{AF}$ episodes within $60 \mathrm{~s}$ in each rat.

\section{Gut Microbiota Profiling.}

The sequencing of $16 \mathrm{~S}$ ribosomal RNA (rRNA)-encoding gene of fecal samples was performed as described previously[15]. Microbiota in fecal samples, representative of that in the proximal colon, was collected from rats and immediately frozen and stored. Bacteria taxa were analyzed by amplifying the $\mathrm{V} 3$ to V4 hypervariable regions of the 16S rRNA gene and sequenced with Illumina HiSeq 2500 platform (Illumina, San Diego, CA). Quality filter of 16S rRNA sequences was conducted by using Quantitative 
Insights Into Microbial Ecology (QIIME) and custom scripts. Resulting sequences were then clustered into Operation Taxonomic Unit (OTU) by searching against Greengenes reference database of 16S rRNA gene sequences and clustered at $97 \%$ by Uclust algorithm[16]. The differences of Genus level and Family level between treatment groups were calculated by using pairwise Wilcoxon rank-sum tests.

\section{Collect Fecal Sample and Microbiota Transplantation.}

Feces from 3 aged rats (22-24 months old) with increased AF susceptibility residing and from young rats (2-3 months old) were collected and the samples were pooled by group. Each feces sample was diluted in sterile PBS (100 mg feces in $2 \mathrm{ml}$ buffer) and homogenized for $5 \mathrm{~min}$. After vortexed for $1 \mathrm{~min}$ and centrifuged at $800 \mathrm{~g}$ for $3 \mathrm{~min}$, the supernatant was collected.

The protocol of fecal microbiota transplantation (FMT) was performed as described by García-Lezana et al.[17] In order to facilitate re-colonization of transplanted gut microbiota, rats were administrated with omeprazole $(50 \mathrm{mg} / \mathrm{Kg} / \mathrm{d})$ for 3 days before FMT for decontamination. To facilitate intestinal emptying, 1 $\mathrm{ml}$ and $2 \mathrm{ml}$ of CitraFleet (sodium picosulfate, $0.16 \mathrm{mg} / \mathrm{ml}$ and magnesium oxide $51.2 \mathrm{mg} / \mathrm{ml}$ ) along with $2 \mathrm{ml}$ of water were administrated to the animals $24 \mathrm{~h}$ and $12 \mathrm{~h}$ prior to FMT, respectively. A study reported by Manichanh et al.[18] showed that the transplanted microbiota can be acquired following a single fecal transplant and can last for at least three months. We therefore adapted a single oral gavage for 6 weeks for re-colonization of transplanted gut microbiota. The young rats gavaged with autogenous fecal were referred to the Young-FMT group and the young rats gavaged with fecal from aged rats to the Aged-FMT group.

For the experiments involving long-term young FMT rescuing study, we collected feces from 3 young without AF rats (2-3 months old). The aged recipient rats (16-18 months old) were administrated by autologous fecal microbiota transplantation (Aged+AgedFMT group) and microbiota transplantation from young rats (Aged+YoungFMT group), respectively, once a week for 6 months.

\section{Nuclear and Cytoplasmic Protein Extraction.}

Pre-frozen atrial tissue was cut into small pieces and washed with PBS, followed by centrifugation at $10000 \mathrm{r} / \mathrm{min}$ for $5 \mathrm{~min}$, according to the subcellular structure nuclear and cytoplasmic protein extraction kit protocol (Wuhan, China). Homogeneous cell suspension was collected by vortex after mixed with cytoplasmic protein extraction reagent $A$ in a tube. Cytoplasmic protein extraction reagent $B$ was then added into the tube on ice. Finally, the tube was centrifuged at $16000 \mathrm{~g}$ for $5 \mathrm{~min}$, and the supernatant was removed, followed by addition of the nucleoprotein extraction reagent to the remaining insoluble cell debris containing nuclei. After $40 \mathrm{~min}$ vortex and $5 \mathrm{~min}$ centrifugation, the supernatant was transferred into a clean tube containing nuclear proteins.

\section{Isolation of Rat Cardiomyocytes.}

Neonatal rat cardiomyocytes (NRCMs) were isolated from 1 to 3-day-old Sprague-Dawley (SD) neonatal rats as previously described[19]. Briefly, neonatal rat hearts were finely minced and placed together in 
$0.25 \%$ trypsin. Cell suspension was collected, centrifuged and re-suspended in DMEM supplemented with $10 \%$ FBS and $100 \mathrm{U} / \mathrm{ml}$ penicillin and $100 \mu \mathrm{g} / \mathrm{ml}$ streptomycin. The re-suspension was plated onto a culture flask, and cardiac fibroblasts (CFs) were obtained after differential adhesion for 90 min leaving cardiac myocytes (CMs) in suspension portion. Then the culture medium was replaced to purify $\mathrm{CFs}$, and the CMs were seeded at a density of $1 \times 10^{6}$ cells per well in a six-well culture plate. Cell cultures were incubated at $37^{\circ} \mathrm{C}$ in a humidified atmosphere with $5 \% \mathrm{CO}_{2}$ and $95 \%$ air. In vitro experiments, the CFs and CMs were cultured with LPS (100 ng/mL)[20] and different concentrations of glucose: $5.5 \mathrm{mmol} / \mathrm{L}$ glucose (control) and $30 \mathrm{mmol} / \mathrm{L}$ glucose $(\mathrm{HG})[21]$ for $72 \mathrm{~h}$ at $37^{\circ} \mathrm{C}$ with $5 \% \mathrm{CO}_{2}$, respectively.

\section{Histology.}

Fresh atrial and proximal colon tissues were cut into $5-\mu \mathrm{m}$ sections, fixed in $4 \%$ paraformaldehyde and embedded with paraffin. Then, the tissues were stained with hematoxylin and eosin (HE), Masson's trichrome and TUNEL staining according to our previous study[22]. The HE staining was used to determine atrial and proximal colon structure. Subsequently, the fibrotic area was measured by Masson's trichrome staining and quantified by using software (Image-pro plus 6.0, Meida Cybernetics LP). Collagen volume fraction was calculated as collagen area/total area $\times 100 \%$. The cell apoptosis was assessed by TUNEL staining and semi-quantified as the number of apoptotic cells per field.

\section{Western Blots (WBs).}

Western blotting procedures in the present study were essentially the same as described in a previous study[22]. Briefly, proteins were separated by electrophoresis on $8 \%$ to $12 \%$ SDS-polyacrylamide gels and transferred moist to polyvinylidene difluoride membranes. The membranes were blocked with $5 \%$ non-fat milk in TBST at room temperature for $1 \mathrm{~h}$ and then incubated with anti-Claudin 4 (Proteintech, 1:500, 16195-1-AP), anti-ZO-1 (Proteintech, 1:500, 21779-1-AP), anti-Occludin (Abcam, 1:1000), anti-TLR4 (Abcam, 1:500, ab1356), anti-NLRP3 (Proteintech, 1:500, 19771-1-AP), anti-Caspase 1 p20 (Invitrogen, 1:200, Prod\#PA5-78915), anti-ASC (Abcam, 1:200, ab175449), anti-IL-1ß (Abcam, 1:500, ab9722), and anti-NF-KB P65 (NOVUS, 1:500, NB100-56721), anti-NF-kB P65 of Phospho-Ser536 (NOVUS, 1:500, NB100-82088), anti-MyD88 (Proteintech, 1:1000, 66660-1-lg), anti-Bcl2 (Abcam, 1:100, ab7973), anti-BAX (Proteintech, 1:500, 60267-1-lg), anti-TGF- $\beta 1$ (Proteintech, 1:1000, 21898-1-AP), anti-a-SMA (Arigo, 1:4000, SQab1735), anti-CD68 (Santa Cruz Biotechnology, 1:100, sc-70761), ISG15 (Abcom, 1:500, ab227541), S100A8 (Proteintech, 1:500, I5792-I-AP), or S100A9 (Proteintech, 1:500, 14226-I-AP), and GAPDH (Zsbio, 1:1000, TA-08) and $\beta$-tublin (CST, 1:1000, \#2148) or $\beta$-actin (Zsbio, 1:1000,TA-09). After washing, the membranes were incubated with the secondary antibody (Santa Cruz Biotechnology, Dallas, USA) for $1 \mathrm{~h}$. The membranes were exposed to ECL buffer after another three washes, and the blots were detected by ChemiDoc XRS gel documentation system (Bio-Rad, Hercules, CA, USA).

\section{FITC-Dextran Permeability Assay.}

Following $6 \mathrm{~h}$ of fasting, rats received a single i.v. dose of $4 \mathrm{kDa}$ FITC-dextran (44mg/ $100 \mathrm{~g}$, Sigma Aldrich, catalog \#FD4). After $4 \mathrm{~h}$ feeding, blood samples wwere collected and centrifuged. FITC fluorescence was 
quantified using the standard curve method according to manufacturer's instructions.

\section{Plasma and Fecal LPS Levels.}

The levels of serum and fecal lipopolysaccharide (LPS) from rats were measured by using a quantitative chromogenic limulus amoebocyte lysate (LAL) QCL-1000 test kit (Lonza Bioscience, Switzerland) according to the manufacturer's instructions. First, the serum and fecal samples were pretreated with pyrogen-free water provided in the kit. Then, the dilute samples were deactivated in $75^{\circ} \mathrm{C}$ water bath for 10 min. After incubation with $50 \mu \mathrm{l}$ of LAL reagent at $37^{\circ} \mathrm{C}$ for $10 \mathrm{~min}, 100 \mu \mathrm{l}$ of LAL chromogenic substrate was added into the samples for $6 \mathrm{~min}$. Finally, $100 \mathrm{mg} / \mathrm{ml}$ of SDS was added to terminate the reaction with yellow color indicating cleavage of the substrate. The OD value of LPS level was measured by spectrophotometrically at $405 \mathrm{~nm}$.

\section{LC-MS/MS Analysis.}

After proximal colon protein extraction, the protein solution was reduced with $5 \mathrm{mM}$ dithiothreitol and 11 $\mathrm{mM}$ iodoacetamide for digestion[23]. The tryptic peptides were dissolved in proper sequence with $0.1 \%$ formic acid (solvent $A$ ) and different concentrations of solvent $B(0.1 \%$ formic acid in $98 \%$ acetonitrile), followed by centrifugation with an EASY-nLC 1000 UPLC system. The peptides were subjected to NSI source followed by tandem mass spectrometry (MS/MS) in Q ExactiveTM Plus (Thermo Fisher Scientific, US) coupled online to the UPLC. Then, the peptides were selected for MS/MS using NCE setting of 28 and the fragments were detected in the Orbitrap. A data-dependent procedure was performed by alternating between one MS scan, which was followed by $20 \mathrm{MS} / \mathrm{MS}$ scans with 15.0 s dynamic exclusion. Automatic gain control (AGC) was set at 5E4. Fixed first mass was set as $100 \mathrm{~m} / \mathrm{z}$.

\section{Statistical Analyses.}

The statistical analyses were performed with GraphPad Prism 6.0 software (GraphPad Software, Inc, La Jolla, CA). Data are all expressed as mean \pm SEM. Two-group comparisons were performed using Student's non-paired $t$-test or Kruskal-Wallis $\mathrm{H}$ test. Data with more than two groups were analyzed by one-way ANOVA, followed by Tukey tests. AF incidence rates were compared with Fisher's exact test. The statistics regarding microbiome analysis in the subsections on microbiome analysis was described in gut microbiota profiling of methods. Differences were considered as statistically significant when $P<0.05$.

\section{Results}

\section{Aging Altered the Gut Microbiota Composition and Promoted AF Susceptibility}

Firstly, we found that the aged rats (22-24 months old) had an increased atrial fibrillation (AF) susceptibility than young rats (2-3 months old) did (Additional File 1:FigureS1A-B) [24, 25], while the atrial effective refractory period (ERP) were no changes (Additional File 1:FigureS1C). To investigate the relationship between gut microbiota and aged-related $A F$, we further profiled the fecal microbiota from aged and young rats by analyzing the DNA sequence encoding the 16S rRNA gene. The PCoA of 
Unweighted UniFrac distances ( $\beta$-diversity) among aged and young rats (Figure1A) showed that microbiota community structures were significantly separated by aging at the first principal coordinate (PC1 axis) and at the second principal coordinate (PC2 axis). On the family level, the abundance of Lactobacillus spp. was higher, whereas the abundances of members in family Bacteroidaceae were clearly lower in young rats than in aged rats (Figure1B). These differences could be traced to a-diversity at both the genus level and operational taxonomic unit (OTU) level (Figure1C), which showed distinct patterns in young as compared to those of aged rats, suggesting a significant shift in gut microbiota composition during aging.

\section{Age-Associated Microbial Transplantation Increased AF Susceptibility and Atrial Fibrosis}

Next, we performed fecal microbiota transplantation (FMT) in young rats by utilizing fecal samples collected from aged AF rats (Aged-FMT) or young rats themselves (Young-FMT) for 6 weeks. Analysis of fecal microbiota composition revealed that aged-related FMT successfully changed the gut microbial ecology of young rats. Unweighted UniFrac distances of 16S rRNA gene sequences showed that the microbiota clustering between Young-FMT group and Aged-FMT group were separated (Additional File 1:FigureS2A). The top 15 differential microbiotas in genus level suggested that the gut microbiota community of recipient rats was successfully transmitted towards to aging phenotype (Additional File 1:FigureS2B). These differences were also traced to abundances of OTUs at the species level (Additional File 1:FigureS2C-D).

Interestingly, burst pacing induced AF in Aged-FMT rats, but not in Young-FMT rats (Figure 1D). Moreover, Aged-FMT had a marked increase in both AF inducibility (Figure 1E) and AF duration (Additional File 1:FigureS2E) as compared with those in Young-FMT rats. However, the AERP were no changes between two groups (Figure 1F). In addition, Masson's trichrome staining showed that atrial fibrosis was markedly increased in Aged-FMT rats (Figure 1G) and the expression levels of TGF- $\beta 1$ and a-SMA in atria, two fibrosis-related marker proteins, were also markedly increased (Figure $1 \mathrm{H}$ ). Taking together, these data suggest that the age-associated microbial dysbiosis causally increased both AF susceptibility and atrial fibrosis.

\section{Aging Human with AF Elevated Circulating LPS Levels}

Gut-derived lipopolysaccharides (LPS) are produced by the outer membrane vesicles of most Gramnegative bacteria and plays an important role in the inflammatory process of aging-related diseases $[5$, 26]. To determine the role of LPS in aging-related $A F$, we collected 1,152 plasma samples of human donors for LPS levels detection. Herein, we found that the plasma LPS concentrations were progressively higher in human donors with advancing age (Figure 2A), while Elderly+AF patients having the highest levels (Figure 2B). Besides, the circulating LPS had good ability (AUC, 0.922; 95\% Cl, 0.867-0.977) to distinguish the elderly patients who presents AF (Figure $2 \mathrm{C}$ ), suggesting that the alteration of circulating LPS during aging had a closely relationship with development of AF.

\section{Inhibition of LPS Prevented Aged Dysbiosis-induced AF Susceptibility and Atrial Fibrosis}


The increased circulating LPS levels were also found in aged rats (Figure 2D) and Aged-FMT rats (Figure $2 \mathrm{E}$ ), suggesting that aged microbiota transfer the LPS of aging phenotype to recipient rats. LPS was exclusively mediated via activation of cell surface TLR4 to trigger MyD88 expression, which leads to the secretion of downstream proinflammatory cytokines[27]. Interestingly, the expression of TLR4/MyD88 pathways were higher in atrial tissue than other organs of aged rats (Figure 2F). Moreover, LPS-RS (1 $\mathrm{mg} / \mathrm{kg}$, once per week for 6 weeks), a potent LPS antagonist, prevented the increased AF inducibility and AF duration in Aged-FMT rats (Figure2G-I). Masson's trichrome staining showed that atrial fibrosis and the expression levels of TGF- $\beta 1$ and a-SMA in atria were also markedly reduced by LPS-RS (Additional File 1:FigureS3A-B). Taking together, these data indicated that aged microbiota could elevate circulating LPS and promoted AF susceptibility.

\section{Age-Associated Microbiota Transplantation Increased Gut Permeability}

There were no differences in the abundance of Gram-negative bacteria and fecal LPS levels between Aged-FMT and Young-FMT (Figure 3A-B), suggesting that the elevated circulating LPS of aging phenotype may be due to the dysfunction of gut barrier. Therefore, we investigated whether the integrity of the intestinal barrier is altered by aged FMT. Firstly, the intestinal permeability was tested with oral fluorescein isothiocyanate (FITC)-dextran. We found that the level of serum FITC-dextran in Aged-FMT rats was significantly higher than that in Young-FMT rats (Figure $3 C$ ). Then, we observed that the levels of tight junction proteins including Zona occluding-1 (ZO-1), occludin (Ocln) and claudin4 (Cldn4), were remarkably decreased in the proximal colon of Aged-FMT rats (Figure 3D). The similar results of tight junction proteins were also found in colon tissues of elderly human (Figure 3E). Moreover, HE and Masson staining of proximal colon revealed a shorter in villi length and an increased fibrotic area in AgedFMT rats (Figure3F and G) and elderly human (Figure3F and H) [28]. Taking together, these observations suggest that loss of integrity of intestinal barrier and alterations of gut pathological are transferable by aged FMT, which may result in transport of a large amount of gut-derived LPS into circulation.

\section{Enhanced Intestinal Inflammation Underlies the Dysfunction of Gut Barrier}

To uncover the mechanisms underlying the microbiota-epithelium crosstalk responsible for the observed gut phenotype, we deeply profiled the proteomics of proximal colon tissue samples. A total of 179 proteins (124 up-regulated and 55 down-regulated) were found to be significantly and aberrantly expressed in proximal colon tissue samples of Young-FMT as compared with those in Aged-FMT (foldchange $>2.0, P<0.05)$. A volcano plots illustrated the profiles of the differentially expressed proteins (Figure 4A) between Young-FMT and Aged-FMT rats. The numbers of differentially expressed proteins were shown in Figure 4B. The Kyoto Encyclopedia of Genes and Genomes (KEGG) pathway analysis of Q1-Q4 proteomics data showed that these differentially expressed proteins were enriched in 33 pathways and that were mainly involved in inflammatory-related pathways (Figure 4 C). So, we further detected the expression levels of representative proteins of enrichment analysis pathways including ISG15, S100A8 and S100A9 of proximal colon tissue in Aged-FMT and Young-FMT rats, to verify the accuracy of proteomic analysis results. The expression levels of inflammation-related markers were remarkably 
increased in Aged-FMT rats (Figure 4D) and elderly human donors (Figure 4E), suggesting a mechanistic explanation for the increased intestinal permeability and the elevated circulating LPS with increased age, which can be transferred by the aged-associated microbial transplantation.

\section{LPS Promoted AF via Activating Atrial NLRP3-Inflammasomes}

LPS-induced activation of TLR4 as priming signal for engaging NF-KB-mediated expression of NLRP3inflammasome, and the later was involved in occurrence of AF[29]. To test whether NLRP3-

inflammasomes was activated in aged dysbiosis-induced AF, we measured protein levels of NLRP3, ASC, and active Casp1-p20 in atria samples collected from elderly patients (over 65 years) who had a history of AF. The expression levels of NLRP3-inflammasomes in atria of Elderly and Elderly+AF patients were markedly elevated, while Elderly+AF patients having the highest levels (Figure 5A), suggesting that NLRP3-inflammasome of aging specific phenotype is related to the pathogenesis of aged-related AF.

Then, we detect the activation of NLRP3-inflammasomes in aged microbiota recipient rats. While protein expression levels of ASC remained unchanged, the levels of NLRP3 and active Casp1-p20 were significantly increased in atria of Aged-FMT rats (Figure5B). Moreover, the upregulation of the NLRP3inflammasome components in Aged-FMT rats was associated with an enhanced activation of TLR4/MyD88/NFKB pathways (Additional File 1:FigureS4A) and an increased nuclear localization of phosphorylated NFKB (pNFkB) (Additional File 1:FigureS4B), suggesting that both "priming" and "assembly" of NLRP3-inflammasome are activated by aged microbiota transplantation. However, the protein levels of CD68, a macrophage marker, were unchanged in both Aged-FMT and Young-FMT groups (Additional File 1:FigureS4C), suggesting that the initial activation of atrial NLRP3-inflammasomes in Aged-FMT rats may be due to the systemic inflammation, but not macrophage infiltration.

Next, we administrated aged microbiota recipient rats with a selective inflammasome-inhibitor MCC950 (15mg/kg i.p. once per week for 6-weeks)[30] to interrupt assembly of the NLRP3-inflammasome complex. Indeed, MCC950 significantly reduced the expression level of atrial NLRP3 pathway in AgedFMT rats (Additional File 1:FigureS5A). Moreover, LPS-RS also significantly reduced activation of atrial NLRP3-inflammasome in aged microbiota recipient rats (Additional File 1:FigureS5B). Burst pacing rarely induced AF in MCC950 rats, whereas it commonly induced AF in Aged-FMT rats (Figure 5C). Both AF inducibility and AF duration were also reduced by MCC950 in Aged-FMT rats (Figure 5D-E). We found that Aged-FMT+MCC950 group rats exhibited less atrial collagen deposition and lower collagen volume fraction (Figure 5F), and lower expression levels of TGF- $\beta$ and a-SMA (Figure 5G). These results suggest that activation of NLRP3-inflammasome is critical for age-associated microbial dysbiosis-induced AF.

\section{LPS and Excess Glucose Synergistically Enhanced NLRP3-Inflammasome Activity}

The NLRP3-inflammasomes were activated in responding to excess extracellular glucose[31] or elevated LPS[32], all of these were accompanied with increased aging[5]. To determine the role of glucose metabolism on aged microbiota-induced AF, the alteration of glucose tolerance both in human and rats were tested. Here, we collected 12,012 oral glucose tolerance test (OGTT) results of clinical individuals 
and found that the plasma glucose levels in responding to OGTT rise consecutively for every decade of age until was peaked at the range of $81 \sim 90$ age (Figure 6A), and the results of peak blood glucose is delayed with increased aging, suggesting that aging leads to prolonged glucose load time. Moreover, the peak glucose levels (Figure 6B) and 2hG levels (Additional File 1:FigureS6A) in responding to OGTT were also progressively higher with aging. Interestingly, $2 \mathrm{hG}$ levels rise with increasing ages in both sexes, reaching higher levels in the men than women with same age (Additional File 1:FigureS6B), which is consistent with the gender difference of AF incidence[33]. The similar trend of aging phenotype still exists in DM and NDM patients, but 2hG levels were always higher in DM than NDM (Additional File 1:FigureS6C). More importantly, we found that the $2 \mathrm{~h}$ OGTT results in Aged-FMT rats were significantly higher than those in Young-FMT rats (Figure6C), while LPR-RS could prevent it (Figure6D). These data suggesting that aged microbiota dysbiosis causes impaired glucose tolerance, and which may result from increased circulating LPS levels.

To explore the underling mechanisms of LPS and high glucose (HG) on atrial NLRP3-inflammasome activation during aging, we assessed the expression levels of NLRP3, ASC, and active Casp1-p20 induced by LPS and HG in vitro. While protein levels of ASC remained unchanged, LPS+HG significantly increased the levels of both NLRP3 and active Casp1-p20 in cardiac fibroblasts (CF) (Figure 6E) and cardiac myocytes (CM) (Additional File 1:FigureS7A), respectively. Interestingly, neither LPS nor HG alone could activate NLRP3-inflammasome in CF, but only when being incubated with both of LPS and HG, can NLRP3-inflammasome be activated (Figure 6E). More importantly, the expression levels of TLR4 and MyD88 were significantly higher in the CF compare with that in CM induced by LPS+HG (Figure 6F), suggesting the TLR4/MyD88 pathways in CF was predispose to LPS and HG. Besides, LPS+HG remarkably increased the expression levels of TGF- $\beta$ and a-SMA in CF (Figure 6G), but not affect the expression levels of apoptosis markers including Bcl-2 and Bax in CM (Additional File 1:FigureS7B). Taken together, these results indicating that LPS and HG synergistically enhance atrial NLRP3inflammasome activity and leads to atrial fibrosis in aged-related AF.

\section{Long-term Young Microbiota Transplantation Prevented Aged-related AF and Atrial Fibrosis}

We next tested the therapeutic potential of microbiota re-colonization on aged-related AF in a long-term FMT rat model. A young microbiota model was established in aged rats (16-18 months old) by FMT for 6 months from young rats (2-3 months old). Firstly, we observed that the expression levels of tight junction proteins were increased in aged recipient rats with young fecal (Aged+YoungFMT) compared with aged recipient rats with autologous fecal (Aged+AgedFMT) (Additional File 1:FigureS8A). In addition, the Aged+YoungFMT rats had a longer villi length in the proximal colon by HE staining (Figure 7A and B). A decrease in fibrotic area of proximal colon was also observed in Aged+YoungFMT rats by Masson staining (Figure 7A and C).

Then, we found that the elevated plasma LPS (Figure 7D) were restored in young microbiota recipient rats. Intriguingly, we found that young FMT significantly decreased activation of atrial NLRP3inflammasomes (Figure 7E). The atrial fibrosis (Figure 7F) and expression levels of TGF- $\beta$ and a-SMA 
(Additional File 1:FigureS8B) were also attenuated by young fecal transplantation. Moreover, the aged rats that re-colonization with young microbiota were failed in inducing AF (Figure 7G) and inducibility (Figure 7H) by burst electrical pacing, as well as the AF duration (Figure 7I), suggesting that long-term young FMT can prevent the occurrence of aged-related AF by inhibiting the activity of atrial NLRP3inflammasome. Taking together, these results further clarify the role of gut microbiota in aged-related AF and provide more pieces of evidence for a therapeutic strategy targeting gut microbiota in aged-related cardiovascular diseases.

\section{Discussion}

The present study provides new pathophysiological insights into the causal relationship between aged gut microbiota and the atrial fibrillation and opens a new venue of therapeutic strategy to treat agedrelated AF (Figure 8). Herein, we showed that age-associated microbial dysbiosis caused gut barrier dysfunction, abnormal elevation of circulating LPS, impairment of glucose tolerance, and enhancement of the activities of atrial NLRP3-inflammasomes, leading to an increase in AF susceptibility. Such a proAF effect elicited by age-associated microbial dysbiosis is reversible as indicated by the abrogation of pro-AF property following long-term young microbiota transplantation. More importantly, the findings at the molecular level in rat models of aging were reproduced with human atrial, colon and plasma samples collected from a large cohort of patients.

Age-associated microbial dysbiosis causes an increased intestinal permeability[28], which in turn promotes the transfer of harmful substances and pathogens to the bloodstream[34]. Herein, we observed that both of aged rats and elderly human donors had reduced expression levels of tight junction (TJs) proteins and increased intestinal permeability. In particularly, by using FMT approach, we found that the gut pathological phenotype including shorter villus and increased fibrosis area of colon were transmitted by aged microbial to young recipient. The pro-inflammatory viral and bacterial pathogens cause typically disruption of TJs and result in dysfunction of gut barrier due to the loss of enterocyte microvilli[35]. Previous studies have shown that the increased Bacteroidetes is associated with dysfunction of innate immune system, resulting in host present in a pro-inflammation state[36], while deficiency of Lactobacillus spp. is involved in gut inflammation and bacterial infection[37]. These results suggest that alteration of microbiota during aging can cause a potent pro-inflammatory effect on aggravating intraintestinal inflammation response, and it may explain why intestinal permeability is increased. More importantly, proteomic analysis showed that inflammation-related signaling in proximal colon was significantly increased in rats received aged microbiota. It could be speculated that if aged microbiota dysbiosis-induced AF is dependent on systemic inflammation, then, there would be some special inducers present in circulation.

The gut microbial metabolites include TMAO and SCFAs and Bile acids and LPS play a pivotal role in the onset and progression of aging-related cardiovascular diseases[3, 38]. LPS was considered as the most potent pathogen association molecular pattern in human that is associated with "inflamm-aging"[5]. Herein, we found that the plasma LPS was increased in aged rats and aged microbiota recipient rats, 
suggesting aged microbiota re-colonization induced circulating LPS. More importantly, we also found that the circulating LPS concentrations were progressively higher with increased age. Aged microbial transplantation successfully transmitted LPS of aging phenotype to young recipient rats, whereas the abundance of Gram-negative bacteria and LPS in fecal were unchanged. These findings confirmed that age-associated microbial elevates circulating LPS by increasing intestinal permeability and resulting host presents in an "inflamm-aging" state. On the other hand, LPS was referred as the potent pathogen association molecular pattern in host, which activated NLRP3-inflammasome through enhancing TLR4/MyD88/NF-KB pathway. Herein, we found that the expression of TLR4 and MyD88 were highest in atria than other organs in aged rats. Thus, we can reason that aged-related AF induced by LPS may depend on the heterogeneity of TLR4/MyD88 in host, and results in serum LPS levels could specificity predicted the disadvantage outcomes in AF patients[4].

Herein, we found that the activity of atrial NLRP3 in elderly patients with or without AF was higher than that in young controls, suggesting that NLRP3 activity is closely associated with AF, especially during aging. Interestingly, inhibition of NLRP3-inflammasome ameliorates the age-related neurodegenerative disease by modulating the relationship between inflammation and gut microbiota composition, suggesting that NLRP3 activity to engaging for dysbiosis-induced aged-related inflammation diseases[39]. As both elevated LPS[5] and hyperglycemia[40] were accompanied by aging, we speculated that both LPS and HG may have promoting effect on aged microbiota-induced AF[4]. Herein, we found that the plasma LPS and glucose in responding to OGTT were increased in aged microbiota recipient. Activation of NLRP3-inflammasome particularly relevant to aging was resulted from a two-step process:

1) "Priming", introducing NLRP3 protein expression triggered by Toll like receptor-NF-kB signaling, and 2) "Assembly", enabling activators to trigger caspase-1 cleavage. LPS recognition by TLR4/MyD88 is achieved by promoting the transfer of NF-KB into cell nucleus where it causes transcriptional activation, resulting in successive activation of NLRP3-inflammasome "priming". Moreover, excess glucose could promote "assembly" of inflammasome complex. Herein, we found that the enhanced NLRP3 was accompanied by an increased expression of TLR4/MyD88/NF-KB pathway in aged-FMT rats, suggesting that both "priming" and "assembly" processes of inflammsome complex are activated. Besides, aged microbiota FMT successfully activated atrial NLRP3-inflammasome and ultimately increased AF susceptibility and atrial fibrosis. In addition, a specific NLRP3-inflammasome inhibitor, MCC950, attenuated AF susceptibility and atrial fibrosis in aged microbiota recipient. Conversely, young microbiota restored the LPS and impaired glucose tolerance in aged rats.

Recent study showed that the enhanced CM-specific NLRP3-inflammasome was involved in development of AF by inducing atrial fibrosis and calcium handling dysregulation[41]. However, whether the LPS and HG had a synergistic effect on aged-related AF due to NLRP3 activation had never been previously reported. In the present study, we found that only simultaneous stimulation with both LPS and HG could enhance NLRP3-inflammasomes in CF. Besides, the expression levels of fibrosis-related proteins in CF were also increased. Moreover, recent study showed that the activation of NLRP3-inflammasome in the hepatic stellate cells (HSCs) due to LPS could promote liver fibrosis also confirmed our findings[42]. The NLRP3-inflammsome is mainly distributed in macrophage and CF, but less in CM[43], and it can interact 
with different types of cells[44]. Thus, we speculated that NLRP3 activation could promote the release of damage associated molecules from $\mathrm{CF}$ into the extracellular space to activate $\mathrm{CM}$ in the development of aged microbiota dysbiosis-induced AF.

Impaired glucose tolerance accompanied with aging is another contributor to development of AF. Here, we collected a total of 12,012 OGTT records of individuals and found OGTT was increased consecutively with aging. Moreover, the peaked levels of $2 \mathrm{hG}$ and $3 \mathrm{hG}$ at range of $81 \sim 90$ ages suggested that glucose loading duration was prolonged by aging. Interestingly, men had a higher $2 \mathrm{hG}$ than women did, which is consistent with the gender difference of AF incidence[33]. Previous study had shown excess LPS led to age-associated hyperglycemia by inducing mitochondrial dysfunction, cellular senescence and adipose tissue dysfunction[45]. Similarly, we speculated that the mechanism underlying aged microbiota-induced increased OGTT is insulin resistance or adipose dysfunction by elevated circulating LPS. Further investigation is required to elucidate the precise mechanisms by which the LPS alter the glucose metabolism during aging.

Our study has several limitations. There is no clear definition of equivalent human microbiota dysbiosis, which yields therapeutic value of FMT based on animal studies[46]. Besides, there is paucity of data on long-term safety of FMT and some clinical case reports showed that FMT treatment causes serious infectious disease[47]. Thus, the safety issue of long-term FMT in elderly AF patients must be carefully evaluated in the clinical settings. Moreover, although the diet and housing conditions were carefully controlled between young rats and aged ones, it the possible influence by unidentified factors on the gut microbiota of aged rats could not be ruled out. Clearly, the mechanisms for the unfavorable alterations of gut microbiota during aging needs further in-depth investigations.

\section{Conclusions}

In summary, in this study we have demonstrated that gut microbiota dysbiosis is associated with the pathogenesis of aged-related AF. Young microbiota transplantation in part attenuated AF susceptibility and atrial fibrosis by inhibition the activity of atrial NLRP3-inflammasome in aged rats. To the best of our knowledge, this study is the first to demonstrate a mechanistic link between age-associated gut microbiota and the pathophysiology of AF. Our results suggest that manipulation of the gut microbiota composition to block activation of NLRP3-inflammasome is a potential novel anti-AF approach, especially in aged subjects.

\section{Abbreviations}

$\mathrm{AF}=$ atrial fibrillation; $\mathrm{ASC}=$ apoptosis-associated speck-like protein; $\mathrm{CF}=$ cardiac fibroblasts; $\mathrm{CM}=$ cardiac myocytes; ERP = effective refractory period; FMT = fecal microbiota transplantation; MyD88 = myeloid differentiation factor 88; NLRP3 = nucleotide binding and oligomerization domain-like receptor family pyrin domain-containing 3; OUT = operational taxonomic unit; TLR4 = toll-like receptor 4 


\section{Declarations}

\section{Acknowledgements}

The authors thank Prof. Baodong Xie and Prof. Guowei Zhang of the cardiac surgeon teams of Harbin for provision of human atrial biopsies. This research has been conducted using resource from the Biobank of First Affiliated Hospital of Xi'an Jiaotong University. We acknowledge Yanan Wang for helpful of discussion. Proteomics services were provided by Jingjie PTM BioLab (Hangzhou) Co.Ltd, Hangzhou, China.

\section{Authors' contributions}

Yun Zhang and Song Zhang performed the statistical analyses, as well as interpreted the results and drafted the manuscript. Yue Li and Yue Wu designed the experiment and helped draft the manuscript. Yingchun Luo; Yongtai Gong; Xuexin Jin; Jiawei Zhang; Yun Zhou; Xiaozhen Zhuo; Zixi Wang; Desen Liang, Shiqi Zhao; Danghui Sun, Dingyu Wang, Wei Xu; Guangjin Qu; Wanlan Bo and Dan Li performed the cells and animal experiments. Xinbo Zhao; Xuejie Han; Yunlong Gao and Hui Yu supervised and performed samples data collection. Yue Li provided comments for the manuscript. The authors read and approved the final manuscript.

\section{Funding}

This work was supported by grants from the State Key Program of National Natural Science Foundation of China (No.81830012 to Yue Li) and National Natural Science Foundation of China (No.81670297 to Yue Li, No.81974024 to Yongtai Gong, No.81822005 and 81970351 to Yue Wu) and the National Key R\&D Program of China (2019YFA0802300 to Yue Wu) and the Clinical Research Award of the First Affiliated Hospital of XJTU (XJTU1AF-CRF-2017-006 to Yue Wu).

\section{Availability of data and materials}

All data generated or analyzed during this study are included in this published article and its supplementary information files.

\section{Ethics approval and consent to participate}

The use of human samples in this study complies with the Declaration of Helsinki and was approved by the Ethics Committee of the First Affiliated Hospital of Harbin Medical University (Ethical approval Number:IRB-AF/SC-04/01.0). The participants all provided a written informed consent. The animal experiments in this study were conducted in accordance with the Guide for the Care and Use of Laboratory Animals and approved by the Institutional Animal Care and Use Committee at the Harbin Medical University (Ethical approval Number: 2019004).

\section{Consent for publication}


Not applicable.

\section{Competing interests}

All authors have no disclosures to declare.

\section{References}

1. Andrade J, Khairy P, Dobrev D, Nattel S. The clinical profile and pathophysiology of atrial fibrillation: relationships among clinical features, epidemiology, and mechanisms. Circ Res. 2014;114:1453-68.

2. Eisen A, Ruff CT, Braunwald E, Nordio F, Corbalán R, Dalby A, et al. Sudden Cardiac Death in Patients With Atrial Fibrillation: Insights From the ENGAGE AF-TIMI 48 Trial. J Am Heart Assoc. 2016;5. https://doi.org/10.1161/JAHA.116.003735.

3. Tang WH, Wang Z, Levison BS, Koeth RA, Britt EB, Fu X, et al. Intestinal microbial metabolism of phosphatidylcholine and cardiovascular risk. N Engl J Med. 2013;368:1575-84.

4. Pastori D, Carnevale R, Nocella C, Novo M, Santulli M, Cammisotto V, et al. Gut-Derived Serum Lipopolysaccharide is Associated With Enhanced Risk of Major Adverse Cardiovascular Events in Atrial Fibrillation: Effect of Adherence to Mediterranean Diet. J Am Heart Assoc. 2017;6. https://doi.org/ 10.1161/JAHA.117.005784.

5. Ghosh S, Lertwattanarak R, Garduño Jde J, Galeana JJ, Li J, Zamarripa F, et al. Elevated muscle TLR4 expression and metabolic endotoxemia in human aging. J Gerontol A Biol Sci Med Sci. 2015;70:232-46.

6. O'Toole PW, Jeffery IB. Gut microbiota and aging. Science. 2015;350:1214-5.

7. Claesson MJ, Jeffery IB, Conde S, Power SE, O'Connor EM, Cusack S, et al. Gut microbiota composition correlates with diet and health in the elderly. Nature. 2012;488:178-84.

8. Peake J, Della Gatta P, Cameron-Smith D. Aging and its effects on inflammation in skeletal muscle at rest and following exercise-induced muscle injury. Am J Physiol Regul Integr Comp Physiol. 2010;298:R1485-95.

9. Guo Y, Lip GY, Apostolakis S. Inflammation in atrial fibrillation. J Am Coll Cardiol. 2012;60:2263-70.

10. Jacob KA, Nathoe HM, Dieleman JM, van Osch D, Kluin J, van Dijk D. Inflammation in new-onset atrial fibrillation after cardiac surgery: a systematic review. Eur J Clin Invest. 2014;44:402-28.

11. Luan Y, Guo Y, Li S, Yu B, Zhu S, Li S, et al. Interleukin-18 among atrial fibrillation patients in the absence of structural heart disease. Europace. 2010;12:1713-8.

12. Zhou SY, Gillilland M 3rd, Wu X, Leelasinjaroen P, Zhang G, Zhou H, et al. FODMAP diet modulates visceral nociception by lipopolysaccharide-mediated intestinal inflammation and barrier dysfunction. J Clin Invest. 2018;128:267-280.

13. Lu YC, Yeh WC, Ohashi PS. LPS/TLR4 signal transduction pathway. Cytokine. 2008;42:145-151.

14. Hiram R, Naud P, Xiong F, Al-U'datt D, Algalarrondo V, Sirois MG, et al. Right Atrial Mechanisms of Atrial Fibrillation in a Rat Model of Right Heart Disease. J Am Coll Cardiol. 2019;74:1332-1347. 
15. Malik M, Suboc TM, Tyagi S, Salzman N, Wang J, Ying R, et al. Lactobacillus plantarum 299v Supplementation Improves Vascular Endothelial Function and Reduces Inflammatory Biomarkers in Men With Stable Coronary Artery Disease. Circ Res. 2018;123:1091-1102.

16. Rühlemann MC, Heinsen FA, Zenouzi R, Lieb W, Franke A, Schramm C. Faecal microbiota profiles as diagnostic biomarkers in primary sclerosing cholangitis. Gut. 2017;66:753-754.

17. García-Lezana T, Raurell I, Bravo M, Torres-Arauz M, Salcedo MT, Santiago A, et al. Restoration of a healthy intestinal microbiota normalizes portal hypertension in a rat model of nonalcoholic steatohepatitis. Hepatology. 2018;67:1485-1498.

18. Manichanh C, Reeder J, Gibert P, Varela E, Llopis M, Antolin M, et al. Reshaping the gut microbiome with bacterial transplantation and antibiotic intake. Genome Res. 2010;20:1411-9.

19. Cordero P, Parikh VN, Chin ET, Erbilgin A, Gloudemans MJ, Shang C, et al. Pathologic gene network rewiring implicates PPP1R3A as a central regulator in pressure overload heart failure. Nat Commun. 2019;10:2760.

20. Muñoz-Rodríguez C, Fernández S, Osorio JM, Olivares F, Anfossi R, Bolivar S, et al. Expression and function of TLR4- induced B1R bradykinin receptor on cardiac fibroblasts. Toxicol Appl Pharmacol. 2018;351:46-56.

21. Pereira MT, Malik M, Nostro JA, Mahler GJ, Musselman LP. Effect of dietary additives on intestinal permeability in both Drosophila and a human cell co-culture. Dis Model Mech. 2018;11. https://doi.org/10.1242/dmm.034520.

22. Liu GZ, Zhang S, Li YY, Liu YW, Zhang Y, Zhao XB, et al. Aldosterone stimulation mediates cardiac metabolism remodeling via Sirt1/AMPK signaling in canine model. Naunyn Schmiedebergs Arch Pharmacol. 2019;392:851-863.

23. Erturk-Hasdemir D, Oh SF, Okan NA, Stefanetti G, Gazzaniga FS, Seeberger PH, et al. Symbionts exploit complex signaling to educate the immune system. Proc Natl Acad Sci U S A. 2019. https://doi.org/10.1073/pnas.1915978116.

24. Henry BL, Gabris B, Li Q, Martin B, Giannini M, Parikh A, et al. Relaxin suppresses atrial fibrillation in aged rats by reversing fibrosis and upregulating $\mathrm{Na}+$ channels. Heart Rhythm. 2016;13:983-91.

25. Hayashi H, Wang C, Miyauchi Y, Omichi C, Pak HN, Zhou S, et al. Aging-related increase to inducible atrial fibrillation in the rat model. J Cardiovasc Electrophysiol. 2002;13:801-8.

26. Vanaja SK, Russo AJ, Behl B, Banerjee I, Yankova M, Deshmukh SD, et al. Bacterial Outer Membrane Vesicles Mediate Cytosolic Localization of LPS and Caspase-11 Activation. Cell. 2016;165:11061119.

27. Pålsson-McDermott EM, O'Neill LA. Signal transduction by the lipopolysaccharide receptor, Toll-like receptor-4. Immunology. 2004;113:153-62.

28. Thevaranjan N, Puchta A, Schulz C, Naidoo A, Szamosi JC, Verschoor CP, et al. Age-Associated Microbial Dysbiosis Promotes Intestinal Permeability, Systemic Inflammation, and Macrophage Dysfunction. Cell Host Microbe. 2017;21:455-466.e4. 
29. Pfalzgraff A, Weindl G. Intracellular Lipopolysaccharide Sensing as a Potential Therapeutic Target for Sepsis. Trends Pharmacol Sci. 2019;40:187-197.

30. Coll RC, Robertson AA, Chae JJ, Higgins SC, Muñoz-Planillo R, Inserra MC, et al. A small-molecule inhibitor of the NLRP3 inflammasome for the treatment of inflammatory diseases. Nat Med. 2015;21:248-55.

31. Wang P, Chen F, Wang W, Zhang XD. Hydrogen Sulfide Attenuates High Glucose-Induced Human Retinal Pigment Epithelial Cell Inflammation by Inhibiting ROS Formation and NLRP3 Inflammasome Activation. Mediators Inflamm. 2019;2019:8908960.

32. Zhang L, Mosoian A, Schwartz ME, Florman SS, Gunasekaran G, Schiano T, et al. HIV infection modulates IL-1 $\beta$ response to LPS stimulation through a TLR4-NLRP3 pathway in human liver macrophages. J Leukoc Biol. 2019;105:783-795.

33. Odening KE, Deiß S, Dilling-Boer D, Didenko M, Eriksson U, Nedios S, et al. Mechanisms of sex differences in atrial fibrillation: role of hormones and differences in electrophysiology, structure, function, and remodelling. Europace. 2019;21:366-376.

34. König J, Wells J, Cani PD, García-Ródenas CL, MacDonald T, Mercenier A, et al. Human Intestinal Barrier Function in Health and Disease. Clin Transl Gastroenterol. 2016;7:e196.

35. Lapointe TK, O'Connor PM, Buret AG. The role of epithelial malfunction in the pathogenesis of enteropathogenic E. coli-induced diarrhea. Lab Invest. 2009;89:964-70.

36. Pahwa R, Balderas M, Jialal I, Chen X, Luna RA, Devaraj S. Gut Microbiome and Inflammation: A Study of Diabetic Inflammasome-Knockout Mice. J Diabetes Res. 2017;2017:6519785.

37. Chen YH, Tsai WH, Wu HY, Chen CY, Yeh WL, Chen YH, et al. Probiotic Lactobacillus spp. act Against Helicobacter pylori-induced Inflammation. J Clin Med. 2019;8. https://doi.org/10.3390/jcm8010090.

38. Zapata HJ, Quagliarello VJ. The microbiota and microbiome in aging: potential implications in health and age-related diseases. J Am Geriatr Soc. 2015;63:776-81.

39. Próchnicki T, Latz E. Inflammasomes on the Crossroads of Innate Immune Recognition and Metabolic Control. Cell Metab. 2017;26:71-93.

40. DeFronzo RA. Glucose intolerance and aging. Diabetes Care. 1981;4:493-501.

41. Yao C, Veleva T, Scott L Jr, Cao S, Li L, Chen G, et al. Enhanced Cardiomyocyte NLRP3 Inflammasome Signaling Promotes Atrial Fibrillation. Circulation. 2018;138:2227-2242.

42. Inzaugarat ME, Johnson CD, Holtmann TM, McGeough MD, Trautwein C, Papouchado BG, et al. NLR Family Pyrin Domain-Containing 3 Inflammasome Activation in Hepatic Stellate Cells Induces Liver Fibrosis in Mice. Hepatology. 2019;69:845-859.

43. Zhang W, Tao A, Lan T, Cepinskas G, Kao R, Martin CM, et al. Carbon monoxide releasing molecule-3 improves myocardial function in mice with sepsis by inhibiting NLRP3 inflammasome activation in cardiac fibroblasts. Basic Res Cardiol. 2017;112:16.

44. Russo HM, Rathkey J, Boyd-Tressler A, Katsnelson MA, Abbott DW, Dubyak GR. Active Caspase-1 Induces Plasma Membrane Pores That Precede Pyroptotic Lysis and Are Blocked by Lanthanides. J 
Immunol. 2016;197:1353-67.

45. Chia CW, Egan JM, Ferrucci L. Age-Related Changes in Glucose Metabolism, Hyperglycemia, and Cardiovascular Risk. Circ Res. 2018;123:886-904.

46. Walter J, Armet AM, Finlay BB, Shanahan F. Establishing or Exaggerating Causality for the Gut Microbiome: Lessons from Human Microbiota-Associated Rodents. Cell. 2020;180:221-232.

47. Carlson PE Jr. Regulatory Considerations for Fecal Microbiota Transplantation Products. Cell Host Microbe. 2020;27:173-175.

\section{Figures}

Figure 1

A

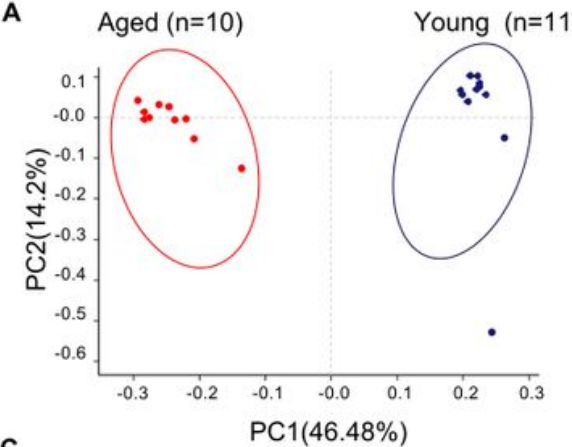

c

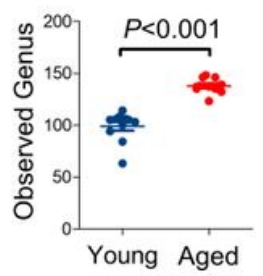

D
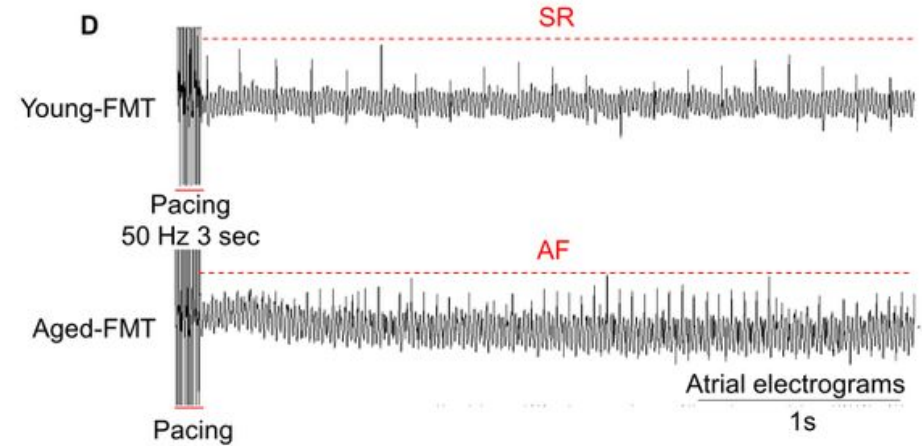

G

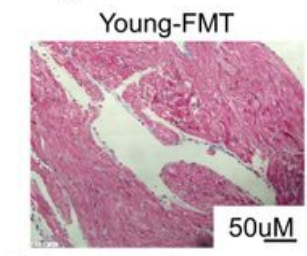

Rats:

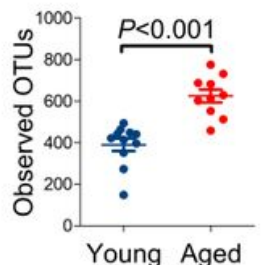

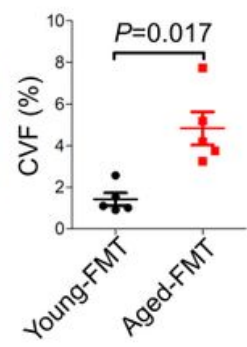

E

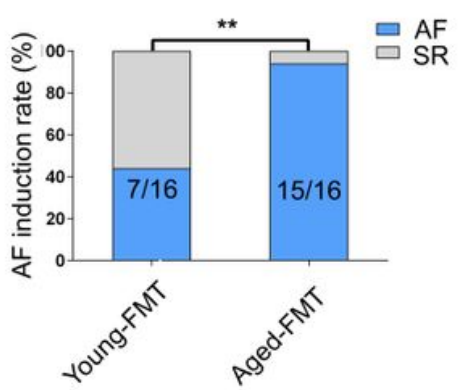

H

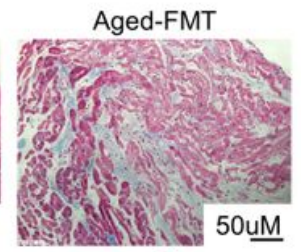

5

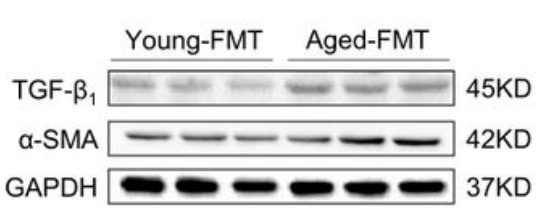

- Young $(n=11)$

- Aged $(n=10)$

Lactobacillaceae Prevotellaceae Ruminococcaceae Lachnospiraceae ales_S24-7_group $\square$

Peptostreptococcaceae Acidaminococcaceae $\mathbf{F}$ Rikenellaceae Christensenellaceae $\mathbf{F}$ Veillonellaceae $\mathrm{P}$ Spirochaetaceae Erysipelotrichaceae Desulfovibrionaceae | Family XIII
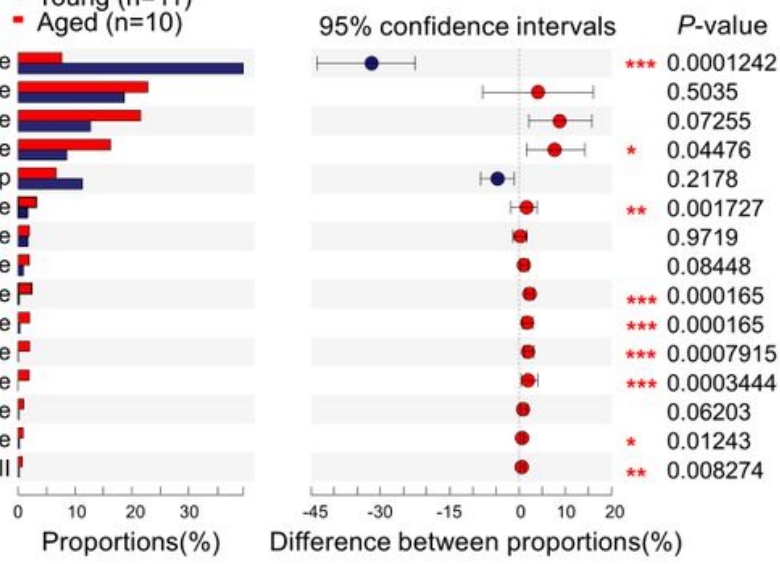

Difference between proportions(\%)

$\mathbf{F}$
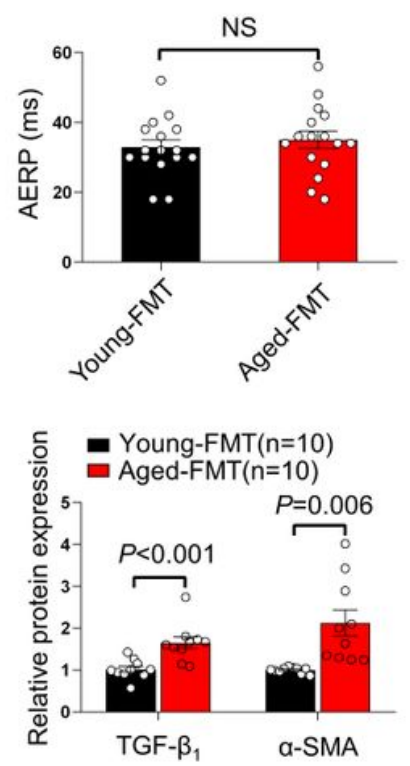

Figure 1 
Aged dysbiosis promotes AF susceptibility and atrial fibrosis. (A) Principal coordinates analysis (PCoA) based on unweighted UniFrac analysis of OTUs. Each symbol represents a single sample of feces. (B) The top 15 of relative abundance of gut microbiota on Family level. (C) The alpha diversity presented at the Genus level or at the OTU level on the basis of 16S-rRNA-encoding gene sequences in feces. (D) Atrial electrograms recordings in response to burst pacing in Young-FMT and Aged-FMT rats. (E) Number of rats in which AF could be reproducibly induced by right atrial burst pacing. (F) The AERP in Young-FMT and Aged-FMT ( $\mathrm{n}=16$ per group) rats. (G) Representative Masson's trichrome staining images and Collagen volume fraction (CVF) in the atria. Scale bar $=50 \mu \mathrm{m}$. (H) Representative WBs and quantification of TGF- $\beta 1$ and $\alpha-S M A$ in atrial tissue. Throughout, data are expressed as mean $\pm S E M .{ }^{*} p<0.05,{ }^{*} p<0.01$; (B) by pairwise Wilcoxon rank-sum test. AF inducibility (E) compared by using the Fisher exact test. AERP, atrial effective refractory period. AF, atrial fibrillation. The $n$ value is showed in each graph.

Figure 2

A

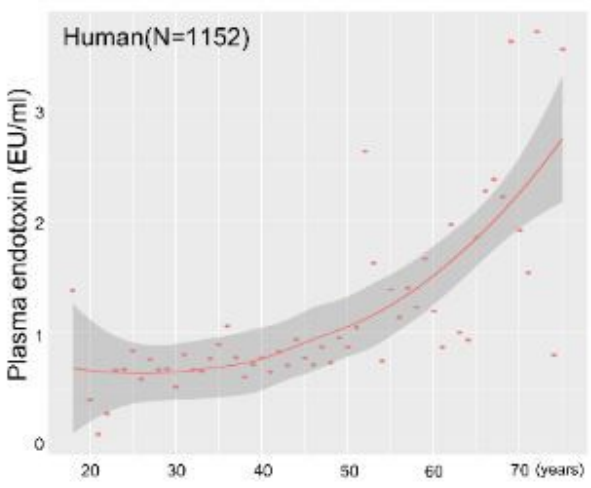

E

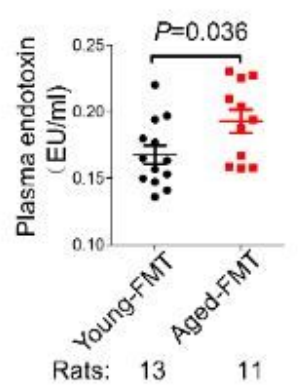

G
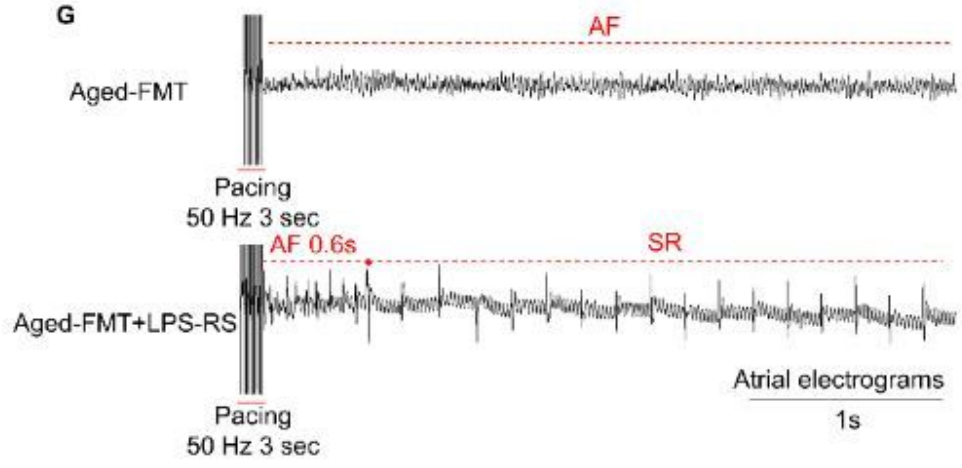

AF

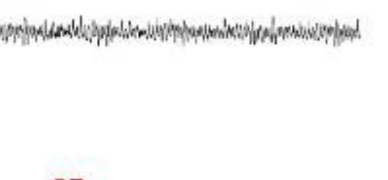

B
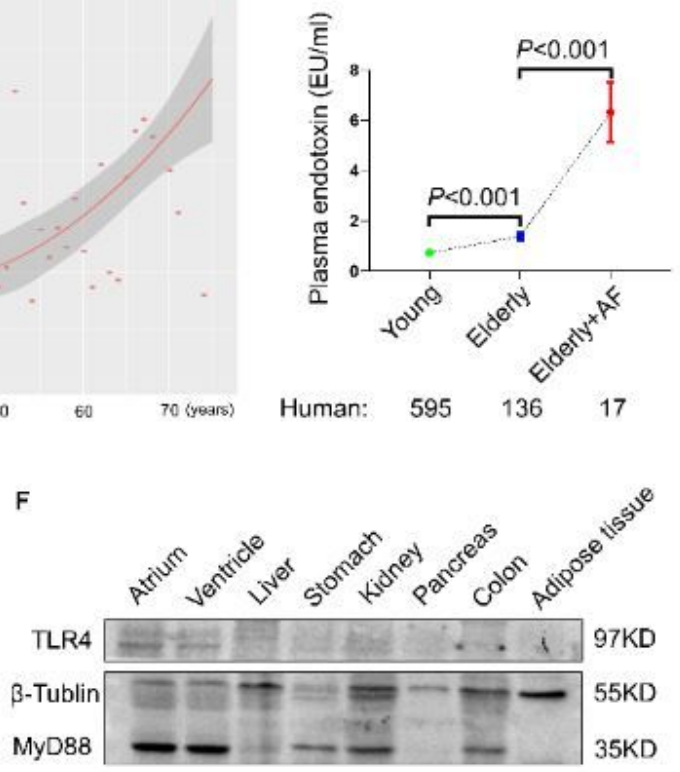

C
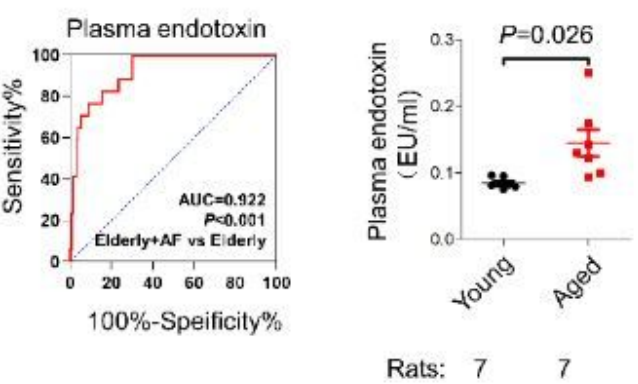

Rats: 77

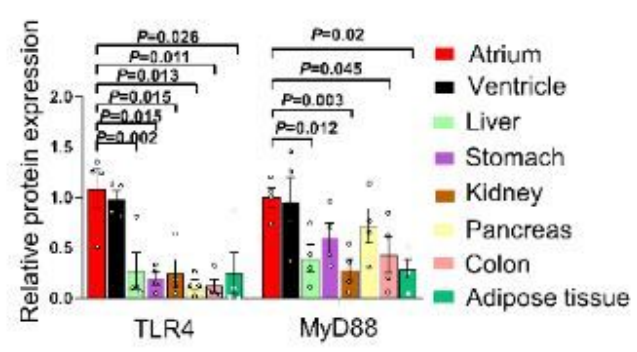


Increased circulating LPS levels during aging promotes AF susceptibility and atrial fibrosis. (A) The results of plasma LPS levels in human donors. (B) The results of plasma LPS levels of elderly patients with or without AF. (C) ROC curves of plasma LPS for predicting AF in elderly human. (D) The results of plasma LPS levels in aged and young rats. (E) The results of plasma LPS levels in Aged-FMT and YoungFMT rats. (F) The representative WBs of TLR4 and MyD88 of different organs in aged rats $(n=4$ per group). LPS-RS reduces AF susceptibility $(G)$ and inducibility $(H)$ and duration $(I)$ by aged FMT $(n=7$ per group). Throughout, data are expressed as mean \pm SEM. ROC, receiver operating characteristic. The $n$ value is showed in each graph.

Figure 3
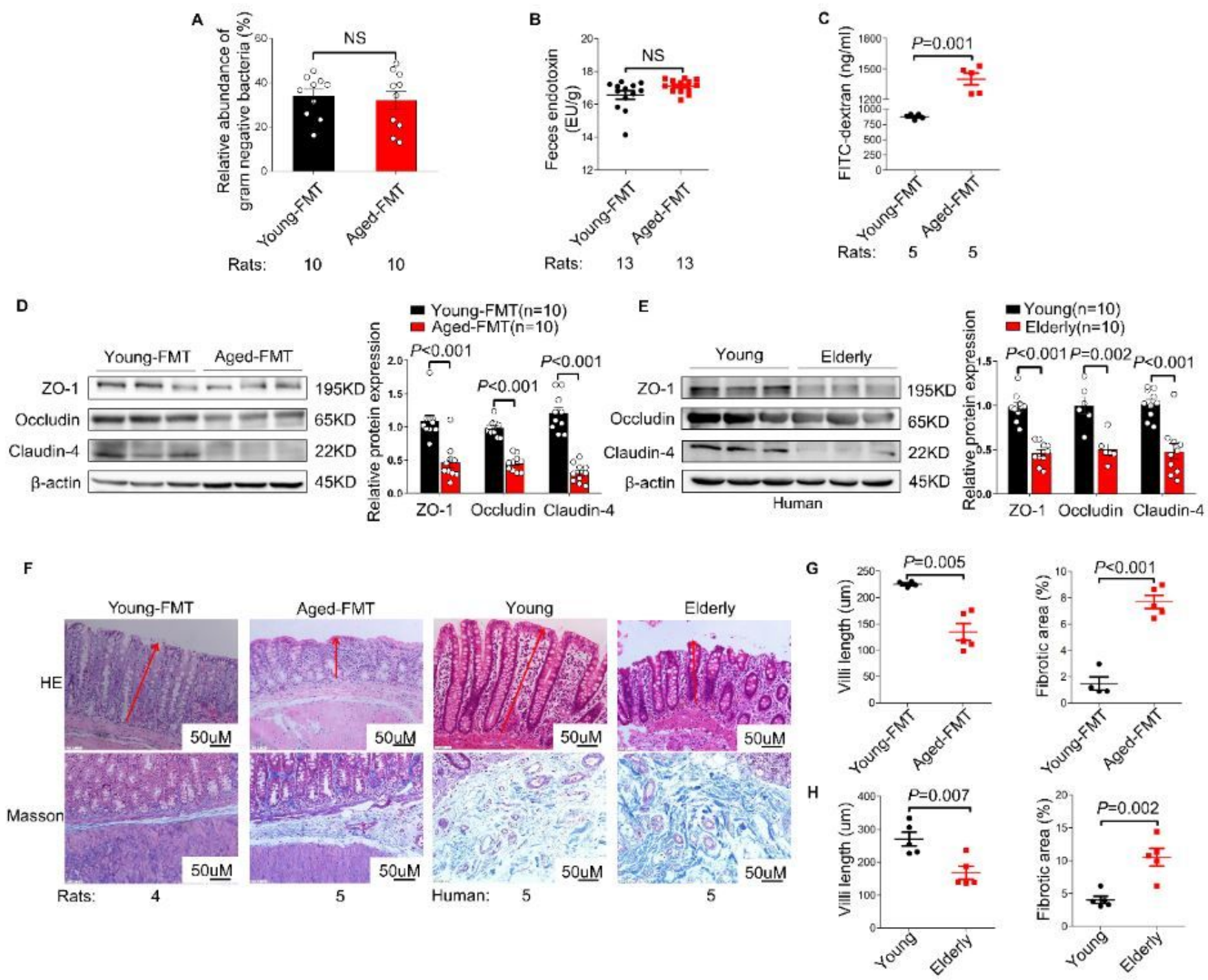

\section{Figure 3}

Aged microbiota transplantation cause gut barrier dysfunction and pathology. The relative abundance of Gram-negative communities (A) and fecal LPS levels (B) in Young-FMT and Aged-FMT rats. (C) Increased fluorescein isothiocyanate (FITC)-dextran concentration in Aged-FMT, but not Young-FMT rats.

Representative WBs and quantification of ZO-1, Occludin and Claudin-4 in proximal colon tissue of rats 
(D) and human donors (E). (F) The examples of HE staining Masson staining measured villi lengths (G) and collagen volume fraction (CVF) $(\mathrm{H})$ of proximal colon samples were analyzed. Data are expressed as mean \pm SEM. Scale bar=50 $\mu \mathrm{m}$. The $\mathrm{n}$ value is showed in each graph.

Figure 4

A

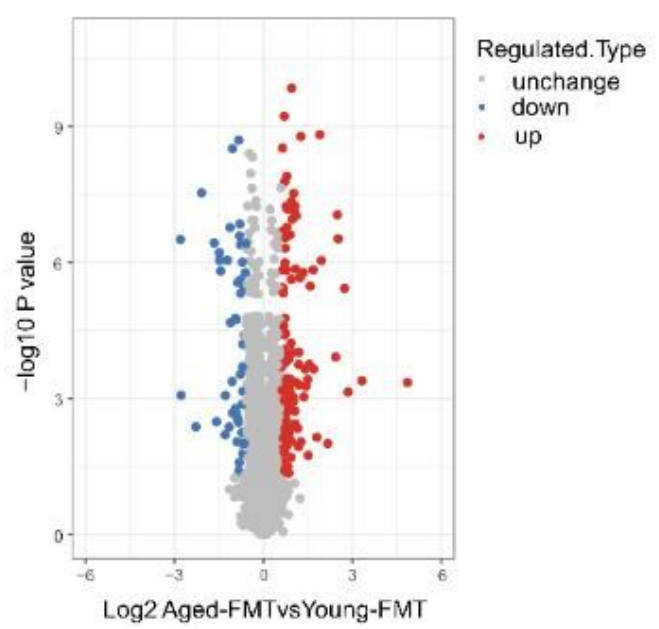

B

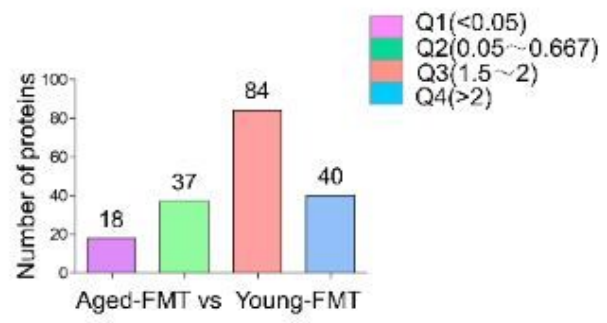

C

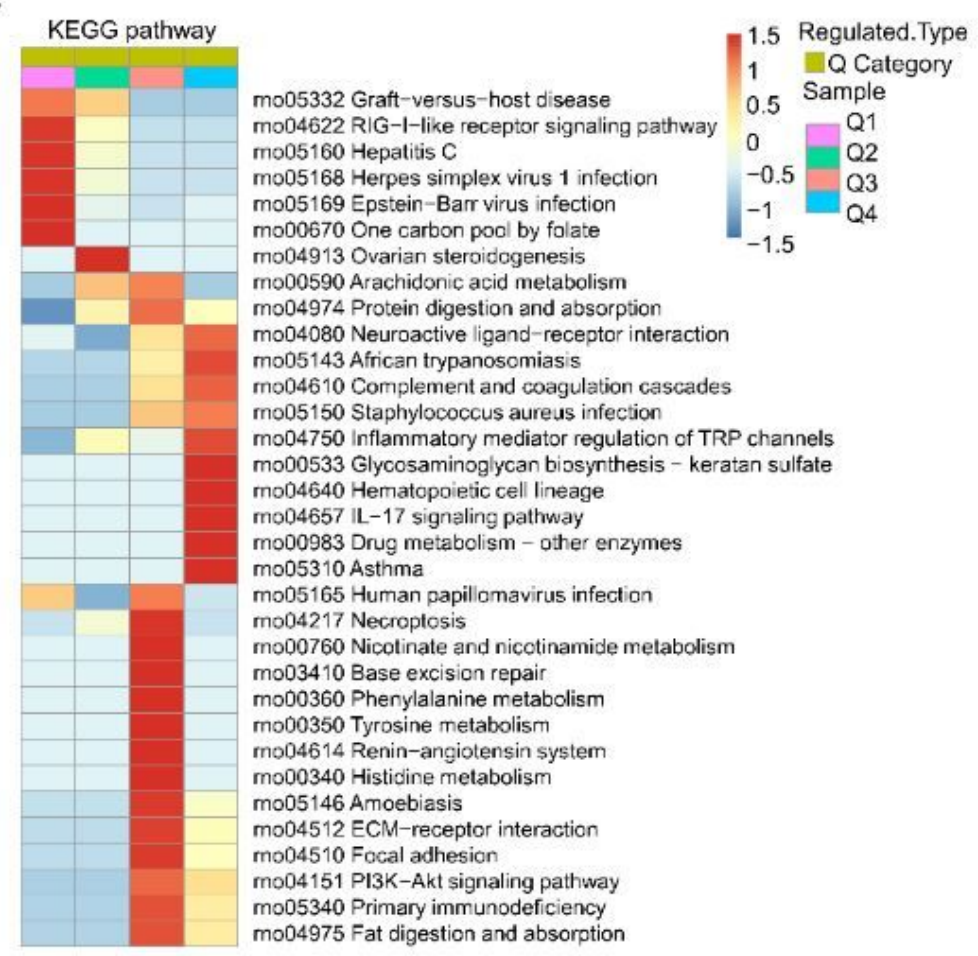

D

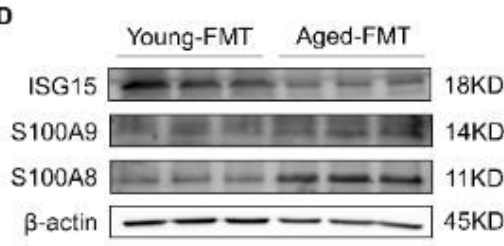

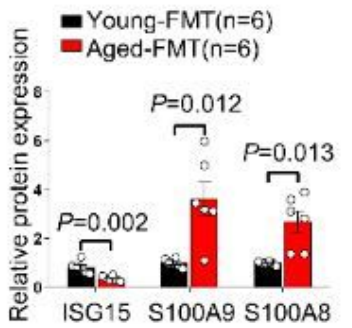

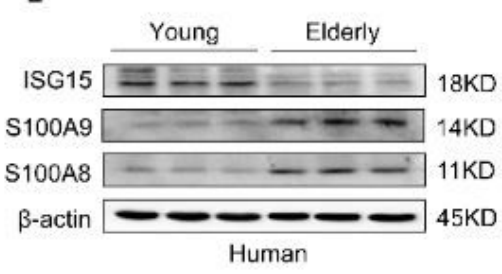

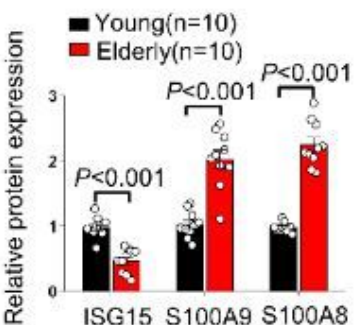

\section{Figure 4}

Aged microbiota transplantation promotes intestinal inflammation. (A) Aberrantly expressed proteins of proximal colon in Aged-FMT and Young-FMT rats ( $n=3$ per group). (B) The numbers of differential expression proteins. (C) KEGG enrichment analysis for all aberrantly expressed proteins. Representative WBs and quantification of ISG15, S100A8 and S100A9 of proximal colon in rats (D) and human donors (E). Data are expressed as meanıSEM. The $n$ value is showed in each graph. 
Figure 5

A
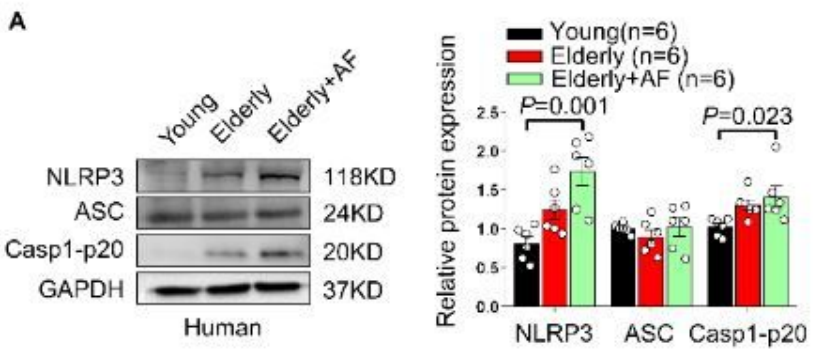

c
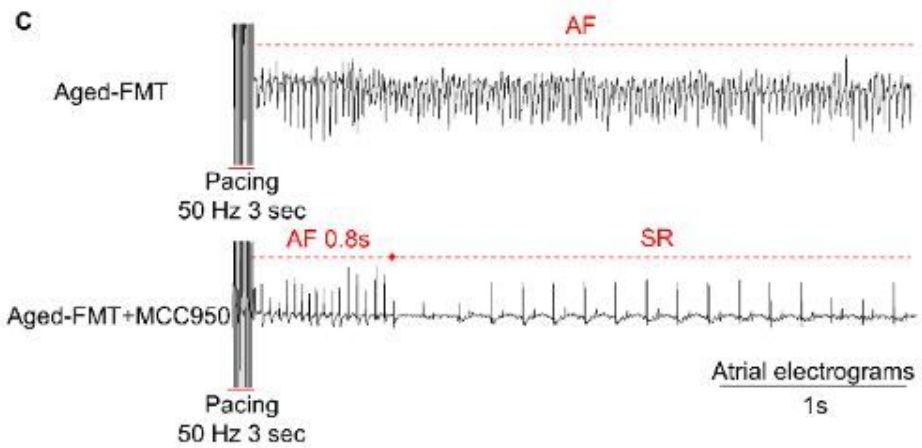

F
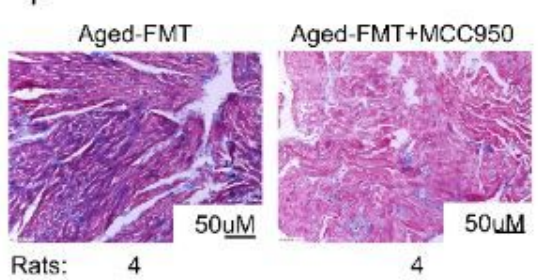

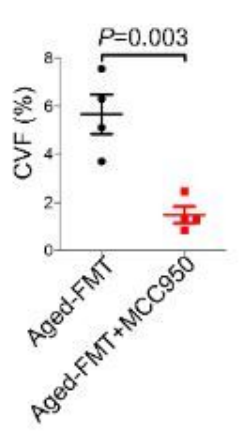

G

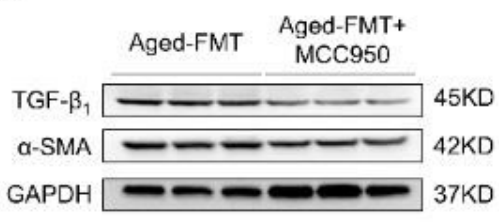

B

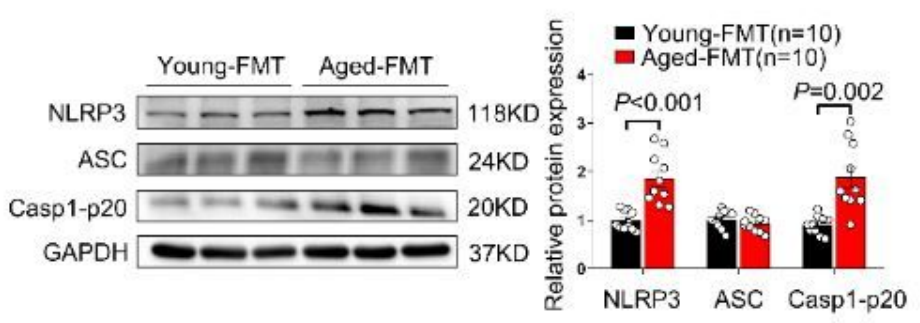

D

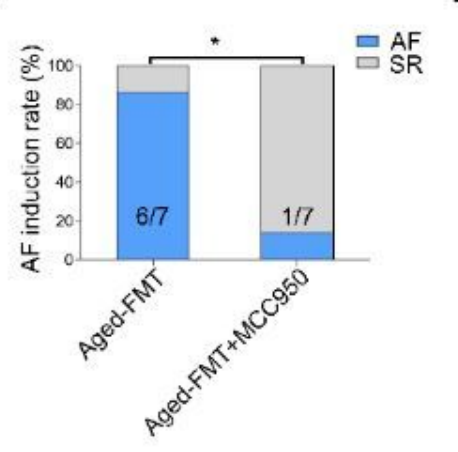

E
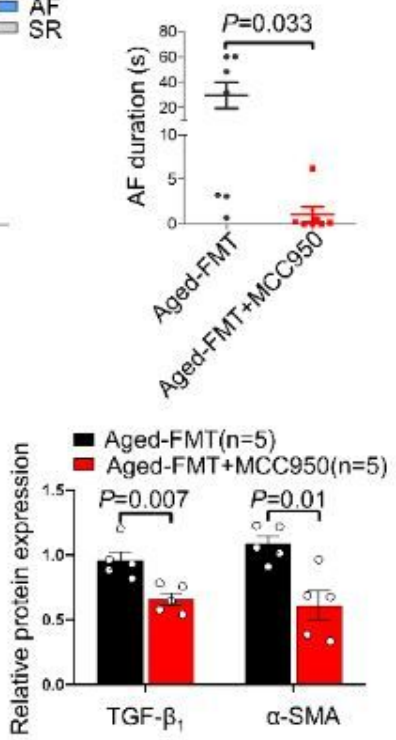

\section{Figure 5}

Aged dysbiosis promotes AF susceptibility by activation of NLRP3 pathway. (A) Representative WBs and quantification of NLRP3, ASC, and Casp1-p20 in atrial tissue of elderly patients with or without AF. (B) Representative WBs and quantification of NLRP3, ASC, and Caspase-1 in atrial tissue of Aged-FMT and Young-FMT rats. (C) Atrial electrograms recordings in response to burst pacing in Aged-FMT and AgedFMT+MCC950 rats. (D) Number of rats in which AF could be reproducibly induced by right atrial burst pacing. (E) The AF duration induced by atrial burst pacing ( $n=7$ per group). (F) Representative Masson's trichrome staining images and collagen volume fraction (CVF) in the atria are shown. Scale bar=50 $\mu \mathrm{m}$. (G) Representative WBs and quantification of TGF- $\beta 1$ and a-SMA in atrial tissue. Data are expressed as mean \pm SEM. ${ }^{*} p<0.05$. AF inducibility (D) compared by using the Fisher exact test. The $n$ value is showed in each graph. 
Figure 6

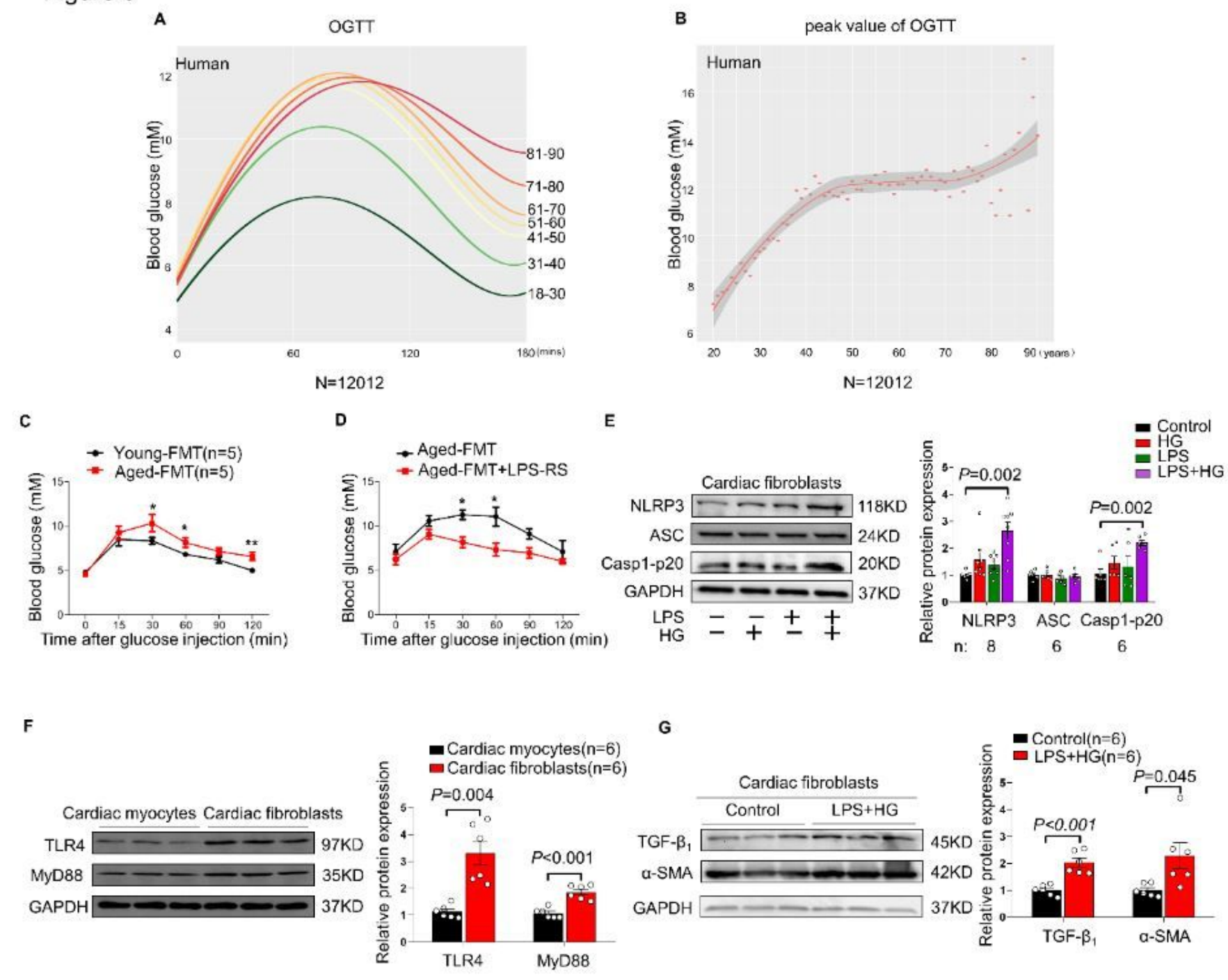

\section{Figure 6}

Aged microbiota transplantation enhanced activation of NLRP3- inflammasome by LPS and high glucose. The 3h results of OGTT (A) and the peak value of OGTT (B) in human donors with each age range. (C) The results of glucose response to OGTT in Young-FMT and Aged-FMT rats. (D) LPS-RS restores impaired glucose tolerance by aged FMT. (E) The synergistic effect on expression of NLRP3 pathways by LPS and HG in cardiac fibroblast. (F) Representative WBs and quantification of TLR4 and MyD88 of cardiac myocytes and cardiac fibroblasts induced by LPS and HG. (G) Representative bands and statistical results for the proteins expression of TGF- $\beta 1$ and a-SMA of cardiac fibroblast with or without LPS+HG. Data are expressed as mean \pm SEM. HG, high glucose. OGTT, oral glucose tolerance test. The $n$ value is showed in each graph. 
Figure 7
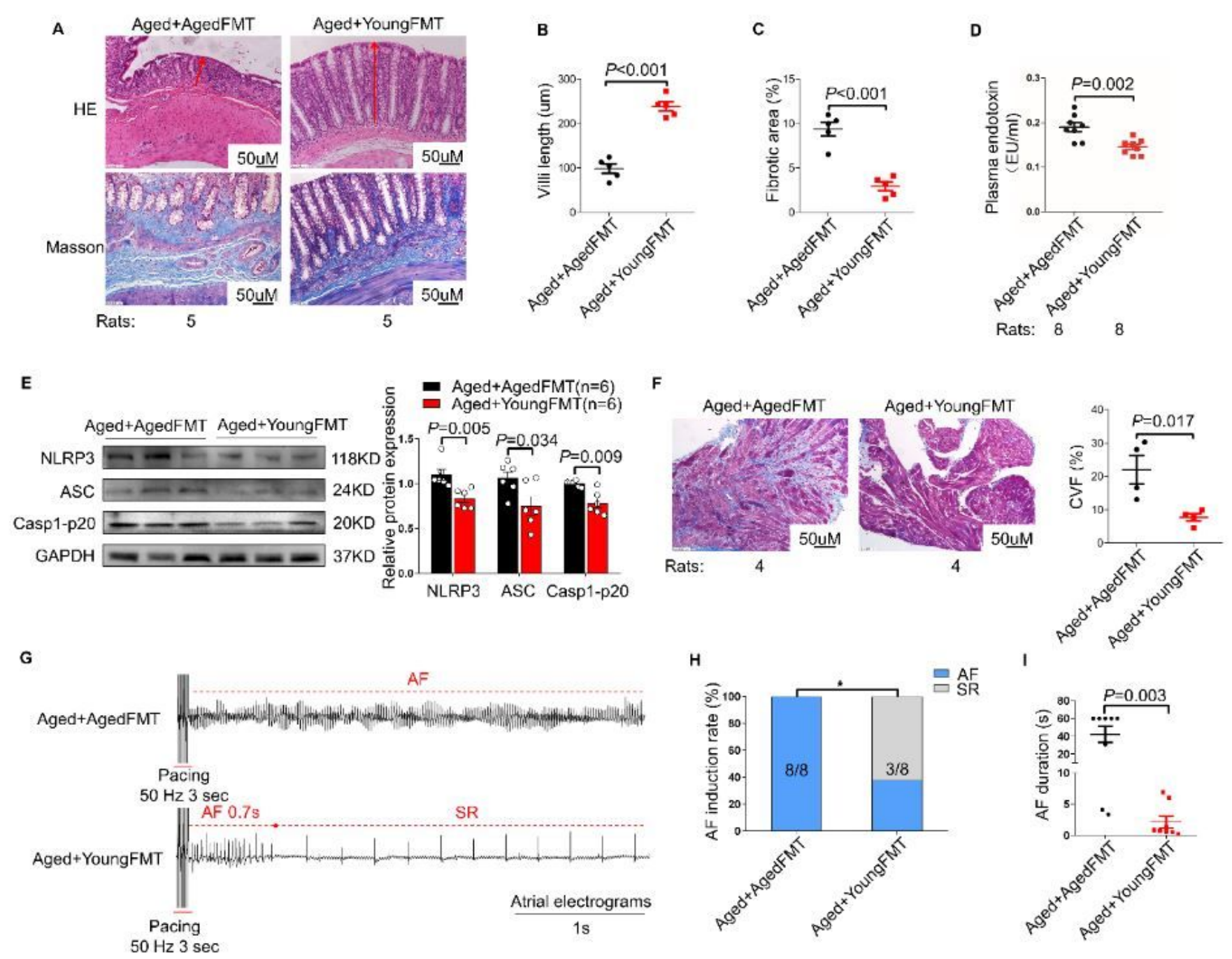

\section{Figure 7}

Young microbiota transplantation prevents AF susceptibility through inhibition of NLRP3-inflammasome signaling pathway and restored gut barrier dysfunction. (A) Examples of HE staining and Masson's trichrome staining of proximal colon samples in Aged+AgedFMT rats and Aged+YoungFMT rats. (B) HE staining shown the villi lengths was shorter in Aged+ AgedFMT rats. (C) Masson's trichrome staining and quantification of colon fibrosis (blue) of Aged+AgedFMT rats and Aged+YoungFMT rats. (D) The results of plasma LPS in Aged+AgedFMT rats and Aged+YoungFMT rats. (E) Representative WBs and quantification of NLRP3, ASC, and Caspase-1 of atrial tissues in all group rats. (F) Masson's trichrome staining images and collagen volume fraction (CVF) of atrial tissues in Aged+AgedFMT rats and Aged+YoungFMT rats. (G) Atrial electrograms recordings in response to burst pacing in Aged+AgedFMT rats and Aged+YoungFMT rats. $(\mathrm{H})$ Number of rats in which AF could be reproducibly induced by right atrial burst pacing. (I) The AF duration induced by atrial burst pacing ( $n=8$ per group). Data are expressed as mean $\pm S E M$. Scale bar $=50 \mu \mathrm{m}$. The $n$ value is showed in each graph. 


\section{Figure 8}

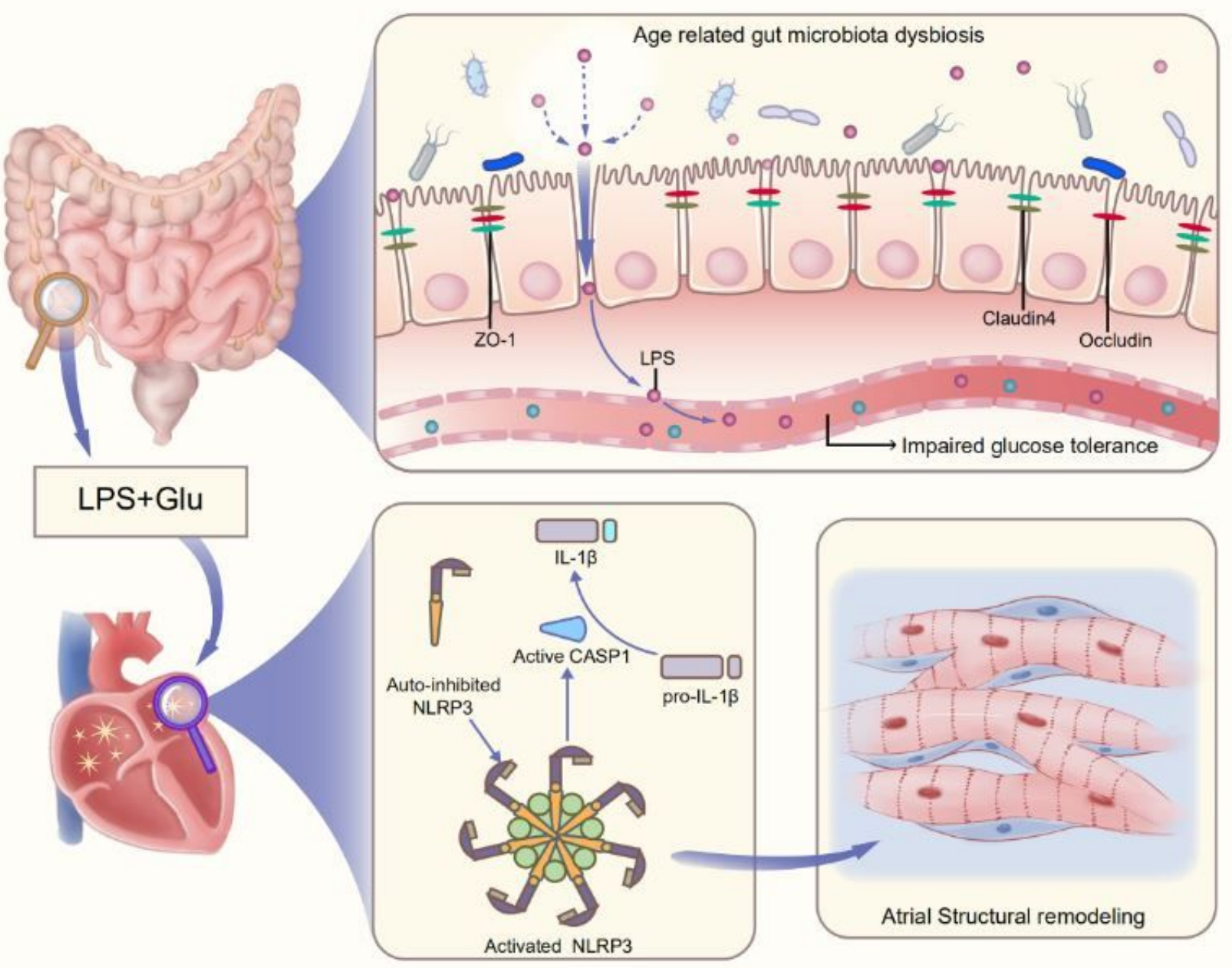

\section{Figure 8}

Working model of AF-promoting mechanisms (bottom left) results from aged microbiota dysbiosis (top left). The consequences of the aged-related gut microbiota dysbiosis are indicated with blue arrows: the dysbiosis lead to reduced expression of tight junction protein including ZO-1 and claudin-4 and occluding in intestinal (top right), which causes increased gut permeability (top right) and leading to LPS transferred into circulation (top right) and may induce impaired glucose tolerance (top right). LPS and excess glucose enhanced atrial NLRP3-inflammasome activity (bottom middle) and that resulting in atrial structural remodeling due to fibrosis (bottom right). LPS, lipopolysaccharide; Glu, glucose; ZO-1, zonula occluden-1; IL-1 $\beta$, interleukin-1 $\beta$.

\section{Supplementary Files}


This is a list of supplementary files associated with this preprint. Click to download.

- Additionfile1.docx 